\title{
Top-Down Feedback Controls the Cortical Representation of Illusory Contours in Mouse Primary Visual Cortex
}

\author{
Alexandr Pak, Esther Ryu, Claudia Li, and $\oplus^{-A l e x a n d e r ~ A . ~ C h u b y k i n ~}$ \\ Department of Biological Sciences, Purdue Institute for Integrative Neuroscience, Purdue University, West Lafayette, Indiana 47907
}

Visual systems have evolved to recognize and extract features from complex scenes using limited sensory information. Contour perception is essential to this process and can occur despite breaks in the continuity of neighboring features. Such robustness of the animal visual system to degraded or occluded shapes may also give rise to an interesting phenomenon of optical illusions. These illusions provide a great opportunity to decipher neural computations underlying contour integration and object detection. Kanizsa illusory contours have been shown to evoke responses in the early visual cortex despite the lack of direct receptive field activation. Recurrent processing between visual areas has been proposed to be involved in this process. However, it is unclear whether higher visual areas directly contribute to the generation of illusory responses in the early visual cortex. Using behavior, in vivo electrophysiology, and optogenetics, we first show that the primary visual cortex (V1) of male mice responds to Kanizsa illusory contours. Responses to Kanizsa illusions emerge later than the responses to the contrast-defined real contours in V1. Second, we demonstrate that illusory responses are orientation-selective. Finally, we show that top-down feedback controls the neural correlates of illusory contour perception in V1. Our results suggest that higher-order visual areas may fill in the missing information in the early visual cortex necessary for illusory contour perception.

Key words: illusion; Kanizsa; optogenetics; silicon probes; top-down feedback; visual cortex

Significance Statement

Perception of the Kanizsa illusory contours is impaired in neurodevelopmental disorders such as schizophrenia, autism, and Williams syndrome. However, the mechanism of the illusory contour perception is poorly understood. Here we describe the behavioral and neural correlates of Kanizsa illusory contours perception in mice, a genetically tractable model system. We show that top-down feedback controls the neural responses to Kanizsa illusion in V1. To our knowledge, this is the first description of the neural correlates of the Kanizsa illusion in mice and the first causal demonstration of their regulation by top-down feedback.

\section{Introduction}

Contours contain essential information about the shapes of objects in the environment. Correct identification of these objects in the visual scene and their segregation from the background are necessary for animal survival. Consequently, the visual system has developed the ability to recognize shapes in diverse conditions, including various levels of illumination, colors, texture, and crowding (Nieder, 2002; Wyatte et al., 2014). One example of this ability is the perception of illusory (or subjective) contours, such as those found in Kanizsa's triangle (Kanizsa, 1976). If three "pacmen" inducers are arranged in a particular configuration,

\footnotetext{
Received Aug. 16, 2019; revised 0ct. 29, 2019; accepted Nov. 15, 2019.

Author contributions: A.P. and A.A.C. designed research; A.P., E.R., and C.L. performed research; A.P. analyzed data; A.P. and A.A.C. wrote the paper.

This work was supported by a Whitehall Foundation Research Grant. We thank Sotiris Masmanidis for providing silicon probes, Scott Pluta and Sam Kissinger for feedback on the paper, and Chubykin laboratory members for useful discussions.

The authors declare no competing financial interests.

Correspondence should be addressed to Alexander A. Chubykin at chubykin@purdue.edu.

https://doi.org/10.1523/JNEUROSCI.1998-19.2019

Copyright $\odot 2020$ the authors
}

one can perceive a triangle despite the lack of a physical basis for that triangle. If these illusory contours are positioned on the receptive field (RF) of a neuron, no neural response is expected because there is no bottom-up input directly activating that RF. However, single-unit recordings in cats and primates have demonstrated that a subset of neurons in early visual cortex responds to subjective contours similarly to real lines (Peterhans and von der Heydt, 1989; von der Heydt and Peterhans, 1989; Grosof et al., 1993; Sheth et al., 1996; Lee and Nguyen, 2001). Importantly, responses in the secondary visual area V2 emerged earlier compared with primary visual cortex (V1; Lee and Nguyen, 2001). Furthermore, recurrent processing is involved in the perception of various illusions across species, suggesting that higher-order processing may be important for the generation of illusory perception (De Weerd et al., 1996; Mendola et al., 1999; Murray et al., 2002; Pan et al., 2012; Luo et al., 2019). These observations led to the hypothesis that top-down feedback from V2 might supply the missing information about subjective contours to V1.

Several recent studies support the importance of top-down feedback in learning and perception (Li et al., 2004; Bar et al., 
2006; Polley et al., 2006; Wyatte et al., 2012; Gilbert and Li, 2013; Schnabel et al., 2018). However, it is poorly understood at the mechanistic level how top-down feedback influences sensory processing in cases where there is no bottom-up input. We decided to test the hypothesis that top-down feedback supplies missing information about subjective contours to $\mathrm{V} 1$ and dissect the neural circuits involved in this process.

Here, we first show that mice can learn to discriminate between Kanizsa illusory contours. Second, mouse V1 responds to Kanizsa illusory contours at the population level. Illusory responses emerge later than the responses to the contrast-defined contours in V1. Third, we demonstrate that illusory responses are orientation selective. Finally, we provide neurophysiological evidence that neural correlates of illusory contour perception can be downregulated by optogenetic inhibition of lateromedial (LM) area.

\section{Materials and Methods}

Mice

All animal procedures were approved by the Purdue University Animal Care and Use Committee. Animals were group-housed on a $12 \mathrm{~h} \mathrm{light/}$ dark cycle with full water and food access. Twelve male C57BL/6 mice (The Jackson Laboratory) aged 2-4 months were used for behavioral studies. Two- to 3-month-old 10 male and 3 female animals were used for optogenetic experiments. Two-month-old five male animals were used for illusory contours orientation tuning experiments.

\section{Initial surgery and viral injections}

Surgical procedures were done as previously described (Kissinger et al., 2018). Briefly, 1-month-old animals were induced with $5 \%$ isoflurane and maintained at $1.5-2 \%$ isoflurane during surgery. They were placed on a motorized stereotaxic apparatus (Neurostar). Animal body temperature was controlled using a heating pad. The scalp was opened to expose the lambda and bregma sutures. A small headpost and a reference pin were installed 3.5 and $0.2 \mathrm{~mm}$ anterior of the bregma, respectively. Neurostar software with an integrated mouse brain atlas was used to mark coordinates above V1 (from lambda, AP: $0.8 \mathrm{~mm}, \mathrm{LM}: \pm 3.1 \mathrm{~mm}$ ) and lateromedial area (from lambda, AP: $1.4 \mathrm{~mm}, \mathrm{LM}: \pm 4.1 \mathrm{~mm}$ ). A small craniotomy was drilled above LM for a viral injection. A glass micropipette was loaded with undiluted AAV5-CAG-ArchT-GFP-WPRE (Addgene, catalog \#29777-AAV5). Injections were performed at $60 \mathrm{nl} / \mathrm{min}$ using Nanoject II (Drummond Scientific) at two depths: 0.7 and $0.3 \mathrm{~mm}$ below the cortical surface. Twenty-five or $50 \mathrm{nl}$ of the virus was injected at each depth and $5 \mathrm{~min}$ was allowed before the glass pipette was withdrawn. We found that both of these concentrations resulted in a localized injection. Medical grade Metabond was then used to seal exposed areas of the scalp to form a head cap. Animals usually recovered within $30 \mathrm{~min}$ after surgery and were followed for $3 \mathrm{~d}$.

\section{Behavioral training paradigm}

Mice were trained to an operant conditioning paradigm through which they learned to discriminate between two visual stimuli, illusory bars in opposite orientations $\left(45\right.$ vs $\left.135^{\circ}\right)$. The mice advanced through a sequence of pre-training, training, and transfer testing sessions, designed for them to progress from simple visual discrimination tasks to more complex ones. The entire training process lasted $\sim 8$ weeks. Training sessions took place in three Sound Attenuation chambers (Lafayette Instrument, series 83017,83018 ) for a duration of $30 \mathrm{~min}$. Each chamber contains a reward tray with an indicator light, a water pump attached to the reward tray (Campden Instruments, Calibrate-able Liquid Pump Model 80204-0.5), and a touchscreen display (Planar, PLL2010MW LED LCD Monitor). ABET II VideoTouch software (Lafayette Instrument) was used to design and execute each stage, and WhiskerServer (Cambridge University Technical Services) was used to set up hardware functionality, e.g., water pump, screen display.

Each mouse was put on water restriction to ensure high motivation during training sessions. Water bottles were removed from the cages, whereas food pellets were available for free feeding. The mice were each given $1000 \mu \mathrm{l}$ of water daily in a small falcon tube cap. Pre-training sessions began once all mice were within $80-85 \%$ of their original weights, which was $7 \mathrm{~d}$ after the start of water restriction. Mice were given a total of $1000 \mu$ l of water daily, so usually, additional water was given following training sessions. Their weights were recorded every day from the start of water restriction to the end of the training, and the amount of water given at the end of a session was adjusted if a mouse was outside of the desired range.

Pre-training. Pre-training consisted of five stages that familiarized the mice with the chamber. The first stage was a free reward stage, where a $160 \mu$ l of water reward was given every $5 \mathrm{~min}$. The reward tray was locked in the open position and the tray light remained on during the entire session. After three sessions of Pre-training Stage 1, mice moved on to Pre-training Stage 2. Mice were given a $20 \mu \mathrm{l}$ reward at the start of the session, and then each time $30 \mathrm{~s}$ after a reward was collected. Rewards were signaled by the reward tray light, and the tray was no longer locked during this stage. Mice passed this stage if they reached at least 20 trials in one session, indicating competence retrieving rewards. All mice passed Pre-training Stage 2 in one session. In each trial of Pre-training Stages 3 , 4 , and 5 , a white square on a black background was displayed to one side of the screen that was pseudorandomly determined. If the side containing the square was touched, it was counted as a correct response. In Pre-training Stage 3, the white square was presented to one side of the screen. After an incorrect response or $30 \mathrm{~s}$ without a response, a $10 \mu \mathrm{l}$ reward was given. After a correct response, a $20 \mu \mathrm{l}$ reward was given to encourage the same behavior. To pass the stage, the mice had to reach at least 20 trials in one session and pass two consecutive sessions. All mice passed Pre-training Stage 3 in 2 sessions. In Pre-training Stage 4, or the Must Touch stage, the white square was presented and remained on the screen until the correct side was touched. Once touched, a $20 \mu \mathrm{l}$ reward was given. Mice passed if they reached at least 20 trials in one session. On average, the mice passed in 1.2 sessions. The last stage of Pre-training was Stage 5, or the Must Initiate stage, where mice had to initiate trials with head entry to the reward tray. Incorrect responses to the blank side prompted a $20 \mathrm{~s}$ timeout and subsequent correction trial, which displayed the image in the same position each trial until a correct response was made. A $20 \mu \mathrm{l}$ reward was given for correct responses, and a $10 \mu \mathrm{l}$ reward was given for correct correction responses. To pass, mice had to reach at least $75 \%$ performance on normal trials, or non-correction trials averaged over 2 consecutive days. On average, the mice took four sessions to pass Pre-training Stage 5. In Pre-training Stages 2, 3, 4, and 5, the intertrial interval (ITI) was $30 \mathrm{~s}$. Once a mouse passed the last Pretraining stage, it proceeded to Training Stage 1.

Training. Training consisted of three stages designed for the mice to learn to discriminate between illusory bars of $45^{\circ}$ and $135^{\circ}$ orientations, where the $135^{\circ}$ bar was the correct image. In each stage, mice were required to initiate trials with a nose-poke to the reward tray, which prompted the images to be displayed in pseudorandom positions. Correct responses were rewarded with $20 \mu \mathrm{l}$ water while correct correction responses were rewarded with $10 \mu \mathrm{l}$. The ITI was $5 \mathrm{~s}$, and incorrect touches prompted a $15 \mathrm{~s}$ timeout. The passing criteria for each stage were at least $75 \%$ performance on normal trials averaged over $2 \mathrm{~d}$, with at least $65 \%$ performance each day. In Stage 1, two black illusory bars were presented, each composed of two white pacmen inducers on a black background. In Stage 2, the illusory bars were presented with two distractor inducers added to each image. Stage 3 used the same stimuli as in Stage 2 and included a color-inverted version, in which the inducers were black and the background was white. The color scheme of each trial was pseudorandom and was not presented more than three times in a row. Once a mouse passed Stage 3, it advanced to the transfer testing stage.

Transfer testing. Stage 4, or the transfer testing stage, incorporated real bar transfer tests into the Stage 3 training trials to test whether the mice had learned to distinguish between the two illusory bars rather than to focus solely on local stimuli. During training trials, stimuli, rewards, ITI, and timeouts were administered exactly as they were in Stage 3. Testing trials were interleaved every five correct responses during normal trials, and two real bars at $45^{\circ}$ and $135^{\circ}$ orientations were presented. Both stimuli positions and color scheme for real bar testing trials were pseudorandomly determined. No reward was given after testing trials, regardless of correct or incorrect responses. Following responses to testing trial stimuli, there was a $5 \mathrm{~s}$ 
ITI before the next training trial. Mice continued in Stage 4 until they accumulated at least 30 testing trials across all sessions.

Control testing. In addition to Stage 4 transfer testing, the mice were also subjected to a control test of differentiating two visual stimuli with rotated distractor inducers. Retaining the Stage 3 training trials exactly as they were, control trials were incorporated after every five correct responses during normal trials. During control trials, the two illusory bars were each shown with two distractor inducers rotated at angles perpendicular to those in Stages 2, 3, and 4. The stimuli positions and color scheme for control trials were pseudorandomly determined.

\section{Pre-recording surgery}

Three weeks after the viral injection, electrophysiological experiments were performed. Two-month-old mice were induced with $5 \%$ isoflurane and placed on the stereotaxic apparatus. Two small craniotomies were performed $<1.5 \%$ isoflurane anesthesia: one above V1 and another one above LM. Mice were then transferred to the recording room and headfixed in the apparatus in front of the monitor.

\section{Electrophysiology}

Animals were habituated in a head-fixation setup for at least $3 \mathrm{~d}$ before the actual experiment. We used 64-channel silicon probes (Shobe et al., 2015; channel separation: vertical $25 \mu \mathrm{m}$, horizontal $20 \mu \mathrm{m}, 3$ columns, $1.05 \mathrm{~mm}$ length) for acute extracellular electrophysiology in awake headfixed mice. The probe was inserted only once per hemisphere so that each animal went through a maximum of two recording sessions. Data acquisition was performed at $30 \mathrm{kHz}$ using OpenEphys hardware and software. Recordings were triggered by a transistor-transistor logic (TTL) signal sent from an Arduino UNO board. PsychoPy software was used to present visual stimuli and trigger both electrophysiology recordings and laser stimulation. This was achieved through serial communication (pyserial package) with Arduino boards. The typical experiment lasted for $\sim 2 \mathrm{~h}$ : $30 \mathrm{~min}$ for the electrode to settle down, 15 min for RF mapping with locally sparse noise, $40 \mathrm{~min}$ for spike detection/sorting, RF analysis, and alignment of RF maps with the Kanizsa figure, and 30 min for recording blocks with the Kanizsa illusion.

\section{Histology}

Mice were anesthetized with $100 \mathrm{mg} / \mathrm{kg}$ ketamine and $16 \mathrm{mg} / \mathrm{kg}$ xylazine solution. They were perfused transcardially with $4 \%$ paraformaldehyde. After decapitation, their brain was extracted and sliced in $0.1 \mathrm{~mm}$ in PBS using a vibratome. Coronal slices were mounted on slides and imaged on a fluorescence scope. We verified the expression of the green fluorescent protein (GFP) in LM (visual lateromedial area in the atlas) but not in V1 by aligning slice images with the mouse atlas (Allen Institute). Histology was performed for all animals to verify GFP expression.

\section{Optogenetic stimulation}

We used a $532 \mathrm{~nm}$ DPSS laser (OEM Laser Systems) for all optogenetic experiments. The light was delivered through an optical fiber $(200 \mu \mathrm{m}$, NA 0.39) coupled to the laser through patch cable (Thorlabs, SMA connection and $1.25 \mathrm{~mm}$ ceramic ferrules at the ends). Laser power was measured using a Powermeter (Thorlabs) before each experiment. We typically used $8-15 \mathrm{~mW}$ of continuous laser stimulation to activate Archaerhodopsin- $\mathrm{T}$ (ArchT) for $0.8 \mathrm{~s}$ starting at $-0.1 \mathrm{~s}$ and ending at $0.7 \mathrm{~s}$ relative to the test stimulus onset. The laser was triggered by a TTL signal from the Arduino board interacting with the running visual stimulation software PsychoPy via serial communication. To protect mouse eyes from laser stimulation and minimize light artifacts on the electrode, we painted optical fibers with black ink and covered the connection between ferrules with foil. Optogenetic trials were interleaved with regular trials.

\section{Visual stimulation}

PsychoPy, open-source Psychology software in Python, was used to generate and present all visual stimulations (Peirce, 2008). We used a gamma calibrated monitor (22 inch, ViewSonic, VX2252, $60 \mathrm{~Hz}$ ) for stimulus presentation. The mean luminance of the monitor was $30 \mathrm{~cd} / \mathrm{m}^{2}$. The monitor was positioned $17 \mathrm{~cm}$ in front of the mouse to ensure the binocular presentation of the stimuli. The size of the illusory and white squares was $44.4^{\circ}$, the width of the black line was $1^{\circ}$, and the radius of the circular disc was $9.2^{\circ}$. There was a $26^{\circ}$ gap between inducers that formed the Kanizsa square illusion. Each trial lasted for $2 \mathrm{~s}$. Each recording session consisted of 200 trials, 4 test stimuli $\times(25$ regular +25 optogenetic) trials. Four circular discs were presented first at $0.5 \mathrm{~s}$, and then they abruptly changed to one of the test stimuli at $1 \mathrm{~s}$. Four test stimuli included a Kanizsa square with illusory contours (KIC), rotated corners control (ROT), a filled white square (SQR), and a black square line (LINE). Each test stimulus was presented for $0.5 \mathrm{~s}$. Stimuli were presented in a pseudorandom manner; optogenetic trials were interleaved with regular trials. Two blocks of 200 trials ( 25 trials for each condition) were presented. The first block was used to pre-expose animals to testing stimuli; both illusory and real squares were shown. Neural responses from the second block were included in the final analysis. For a RF mapping, we used a locally sparse noise with $3.55^{\circ}$ black/white squares (Zhuang et al., 2017). In total, 3002 different frames were presented to the animal. Each frame contained 3-5 black/white squares and lasted for $150 \mathrm{~ms}$. After recording, spike detection and sorting were performed (see Analysis of units). We then computed the spike-triggered average (STA) map for each recorded neuron. A putative RF map was identified by finding the hotspot in the map. After that, we manually aligned the Kanizsa square with RF maps in Adobe Illustrator, so that the illusory contour was on the RF, but inducers were outside it. We tried different coordinates for the Kanizsa square presentation in PsychoPy and used the one that aligned with the majority of units. Depending on the location of the RF, we positioned subjective contours either in a horizontal or vertical orientation, which is different from the previous single-unit studies (KIC of preferred orientation was presented). This was done because we recorded many units at the same time and could not adjust the orientation and position for each cell.

To test the orientation selectivity of KIC, we made several adjustments to our experimental design. First, we used a bigger monitor (27 inch, ViewSonic, VA2746, $60 \mathrm{~Hz}$ ) with $1920 \times 1080$ resolution binocularly positioned at $17 \mathrm{~cm}$ viewing distance. Second, we used $3.96^{\circ}$ black/white squares for RF mapping. The radius of inducers was $6.6^{\circ}$ and the square was $33 \times 33^{\circ}$. There was a $20^{\circ}$ gap between inducers. The line width was $0.83^{\circ}$. Third, we recorded eight drifting gratings before KIC sessions to obtain direction-tuning curves. KIC of four different orientations $\left(0,45,90\right.$, and $\left.135^{\circ}\right)$ were presented to the animal. Overall, we had 16 different stimuli ( 4 test stimuli $\times 4$ different orientations). Test stimuli were presented in a pseudorandom manner, each stimulus was presented for at least 25 trials.

We generally observed a good correspondence of RF positions across cortical depth (see Fig. 3). To quantify the size of the receptive fields, we performed a thresholding of STA RF maps and then fit a twodimensional (2D) Gaussian function (see Fig. 3). Thresholding was done by identifying outlier pixels that were brighter/darker (On/Off) than $97.5 \%$ of pixels within the map. All other pixels were masked and not considered for further analysis. A 2D Gaussian was fitted with optimize.curvefit function from the Python Scipy library. Starting parameters such as $x-y$ position of the center and amplitude were calculated from the thresholded map, whereas initial $\sigma$ was set to 20 . RF size was calculated by averaging a half-width at half-maximum of two axes of the 2D Gaussian fit. Depending on the recording, we were able to obtain On RF maps for $\sim 50 \%$ and Off maps for $12 \%$ of neurons. Given the size of the squares used in the RF mapping, it is possible that they best worked for obtaining RF maps of particular units. As it was reported previously, there is a big diversity in the shape and size of RF maps of mouse V1 neurons (Niell and Stryker, 2008).

\section{Analysis of units}

We used Kilosort software for spike detection and sorting. It implements a template matching algorithm written in MATLAB that allows GPU acceleration (Pachitariu et al., 2016). Speed and quality of the spike sorting were important for our experimental design because the RF mapping and analysis were a critical step to properly position the Kanizsa square. We used default configuration parameters but the SD for spike detection was changed from -4 to -6 . Templates were initialized from the data. Kilosort was run on NVIDIA GeForce GTX960 on Windows 10 running machine. Results were visualized with Klusta/Phy software to manually 
remove, split, and merge units (Rossant et al., 2016). Units were excluded from the further analysis in case they had $<100$ spikes for each testing condition, $>5 \%$ of spikes violated absolute refractory period, aberrantly shaped waveform. Splitting and merging required more manual curation, which was done according to the guide available online (https:// github.com/kwikteam/phy-contrib/blob/master/docs/template-gui.md). We also used Kilosort2 for a subset of data. Both algorithms gave qualitatively the same result, but Kilosort2 required less manual curation. RF mapping and the Kanizsa figure recording blocks were concatenated and clustered together so that we were able to track single units across time. All further analysis was done in Python 2.7 using custom written Jupyter Notebooks and publicly available packages including numpy, pandas, scipy, and seaborn. Peristimulus time histograms (PSTHs) were generated by binning spike times with a $10 \mathrm{~ms}$ window and convolving with a Gaussian kernel (width $100 \mathrm{~ms}) . Z$-score $[z=(\mathrm{FR}-$ mean FR)/SD FR], where FR represents a firing rate during the whole trial, so that mean and SD were computed for the whole $2 \mathrm{~s}$ period of the trial. We computed a response modulation indices for KIC: $I_{\mathrm{m}}(X)=($ FR KIC - FR $X) /($ FR KIC + FR $X)$, where $X$ is firing rate (FR) to the circular discs $-I_{\mathrm{m}}(\mathrm{CIR})$, rotated corners $-I_{\mathrm{m}}(\mathrm{ROT})$, or to the subjective contours during the laser stimulation $-I_{\mathrm{m}}$ (laser). Mean FR was calculated by averaging a response between 50 and $500 \mathrm{~ms}$ after stimulus onset. These indices range from -1 to 1 with positive values indicating a stronger response to the illusory contours and negative ones vice versa. To identify illusory responsive units, we first found units that were not significantly modulated by CIR. We used the Wilcoxon signed rank test to compare baseline firing rate $(0.05-0.5 \mathrm{~s})$ versus CIR $(0.55-1 \mathrm{~s})$ response during KIC trials. Second, we used selected units with positive $I_{\mathrm{m}}(\mathrm{CIR})$ indices. This was done to remove CIR responsive units that were not eliminated by the first criteria. Careful examination of those units revealed that they initially increased their response to CIR (50-200 ms) and then their firing rate dropped below the baseline activity (200-500 ms). This is why their CIR response was not significantly different from the baseline. For experiments with KIC of different orientations, we selected units that had minimal response to CIR in at least two orientations and were illusory responsive. Given that KIC position was not adjusted separately for every unit, it was challenging to find units that did not respond to CIR in all four orientations. This is why we allowed small CIR responses in a subset of conditions. To adjust for these CIR responses, we used $I_{\mathrm{m}}(\mathrm{CIR})$ modulation indices that represent test stimuli responses normalized by CIR response.

For a direction tuning using drifting grating stimuli, we averaged responses for each direction between 0.35 and $0.8 \mathrm{~s}$. For the orientation tuning, responses were averaged between opposite directions. To construct a population-tuning curve, we first identified a preferred orientation (the one with the maximal response). After that, we averaged tuning curves aligned by their preferred orientation, so that $0^{\circ}$ represents the responses to the preferred orientation. All other orientations were then presented relative to the preferred one. To compute putative population tuning curves for KIC and other test stimuli, we used $I_{\mathrm{m}}(\mathrm{CIR})$ to adjust for possible direct RF activation in a subset of conditions. $I_{\mathrm{m}}(\mathrm{CIR})$ was computed separately for each test stimuli and represents normalized to CIR response. For example, $I_{\mathrm{m}}(\mathrm{CIR})$ for SQR would show how much SQR was upregulated relative to the CIR preceding it. We only included conditions with positive $I_{\mathrm{m}}(\mathrm{CIR})$ values. We averaged putative test stimuli tuning curves aligned on the maximal response. Other orientations were relative to the one with the maximal response.

\section{Statistical analysis}

All statistical tests were performed in Python using scipy.stats. Data were not checked for normality of residuals and only non-parametric tests were used. Kruskal-Wallis test was used to determine whether at least one group median is different from others. It was used to test whether population orientation tuning curves were significantly different. Pairwise Mann-Whitney $U$ tests were used to compare the firing rate to illusory contours versus rotated corners, white square, and black line square. Kolmogorov-Smirnov two-sample tests were used to compare the distribution of peak times of units, cortical depth, and RF sizes. We used the Wilcoxon signed rank test to compare the firing rate in response to test stimuli with versus without LM inhibition.
Data and code availability

The data that support the results of the current study and the analysis code are available from the corresponding author upon reasonable request.

\section{Results}

\section{Behavioral correlates of illusory contour perception in mice}

Illusory perception has been described in various species, including insects, birds, and fish (Nieder, 2002). Surprisingly, there are only a couple of studies of illusory perception in rodents (Kanizsa et al., 1993; Okuyama-Uchimura and Komai, 2016). A recent report suggests that mice can discriminate illusory bars in a touchscreen-based visual discrimination task (Okuyama-Uchimura and Komai, 2016). Animals were first trained to discriminate between real bars of different orientations in an operant conditioning chamber. After several training stages, they were exposed to transfer trials in which Kanizsa-type illusory bars were presented. Mice were able to discriminate the illusory bars formed by pacmen inducers.

We decided to test whether mice can learn to discriminate between illusory bars of different orientations. Using a similar touchscreen operant conditioning chamber, we trained mice to discriminate between illusory bars formed by two pacmen inducers (Fig. 1A; Horner et al., 2013). We then performed a reverse transfer experiment to test whether they can differentiate between two real bars (formed by the luminance contours) that they were not explicitly trained on. Our training paradigm consisted of pre-training and four training stages. Pre-training included habituation of water-restricted animals to the behavioral apparatus. During the first training stage, mice were trained to discriminate between two Kanizsa illusory bars ( 45 vs $135^{\circ}$ ) formed by pacmen. We then added two additional pacmen, to ensure that animals were not relying on the local configuration of pacmen. During the third stage, we added color inverted versions of our stimuli to equalize luminance distribution across stimuli (Fig. $1 B)$. During the Test Stage 1, trials with illusory and real bars were interleaved. During the Test Stage 2, we presented illusory bars with rotated distractor pacmen that the animals have not previously seen, to control for the local pacmen inducer configurations. On average, it took animals $7.85 \mathrm{~d}$ to pass the Stage 1 , which required an average performance of 0.75 over the last $2 \mathrm{~d}$ (Fig. 1D). Stages 2 and 3 took 5.71 and $6.42 \mathrm{~d}$ to pass, respectively. Seven of 12 animals were able to pass to the test stages. We found that animals had a high performance in normal trials with illusory bars throughout both test stages [Fig. $1 E$; mean \pm SEM, performance Test 1: $0.76 \pm 0.02(t=10.7, p=3.92 \mathrm{E}-5)$, Test 2: $0.77 \pm 0.04(t=6.3, p=0.001)$, one-sample $t$ test against a chance level of $0.5, n=7$ and 5 mice) $]$. They also had a significantly higher than chance performance in the transfer testing trials with real bars [Fig. $1 E$, left; mean \pm SEM, performance: $0.63 \pm 0.04(t=3.03, p=0.02)$, one-sample $t$ test against a chance level of $0.5, n=7$ ] and rotated distractor pacmen [Fig. $1 E$, right; $0.65 \pm 0.03(t=4.3, p=0.01)$, one-sample $t$ test against a chance level of $0.5, n=5)$. These results suggest that animals were able to learn to differentiate between illusory bars. In particular, their ability to discriminate real bars strongly supports the idea that mice have learned to differentiate between illusory bars rather than the local features in the stimuli. Our results provide additional behavioral evidence for illusory contour perception in mice.

Neural correlates of illusory contour perception in mouse V1 To determine whether mouse V1 responds to subjective contours, we performed extracellular electrophysiology with 64channel silicon probes (Shobe et al., 2015; Fig. 2A,B). We used a 
A

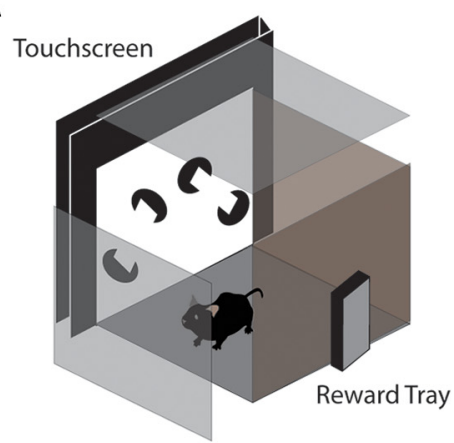

C Test stage $\left\{\begin{array}{l}\text { Normal trials } \\ \text { Test trials }\end{array}\right.$ Normal trials $(\mathrm{N})$

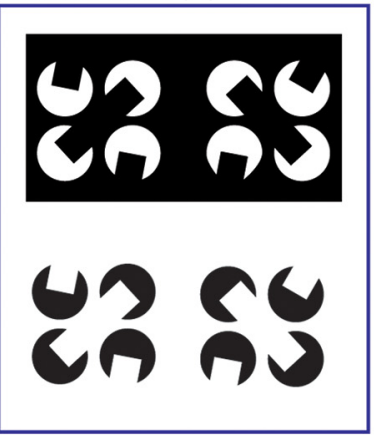

D Stage 1

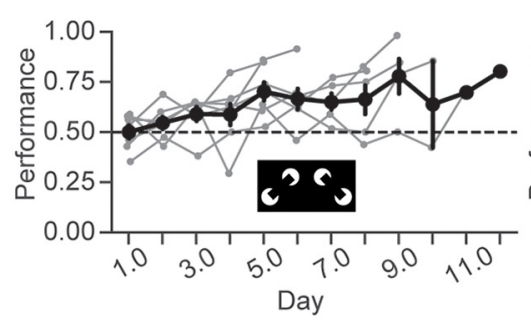

B

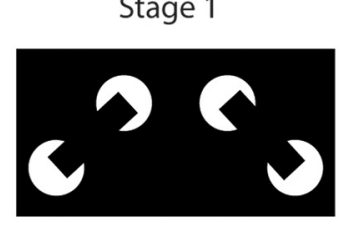

Stage 3

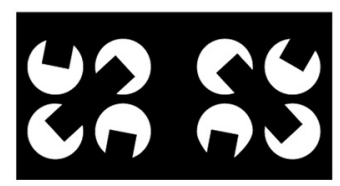

Test 1: real bars (T1)

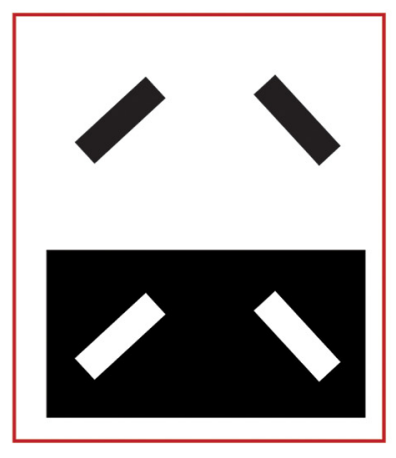

E Test stage

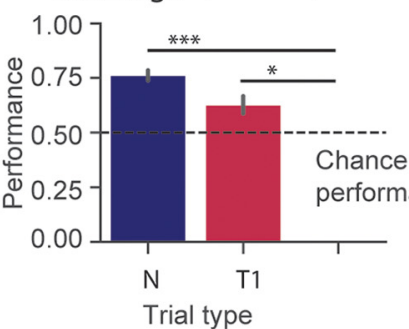

Stage 2
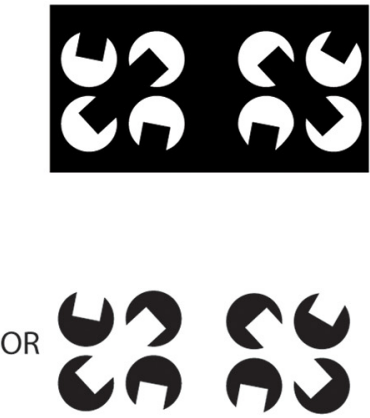

Test 2: rotated side pacmen (T2)
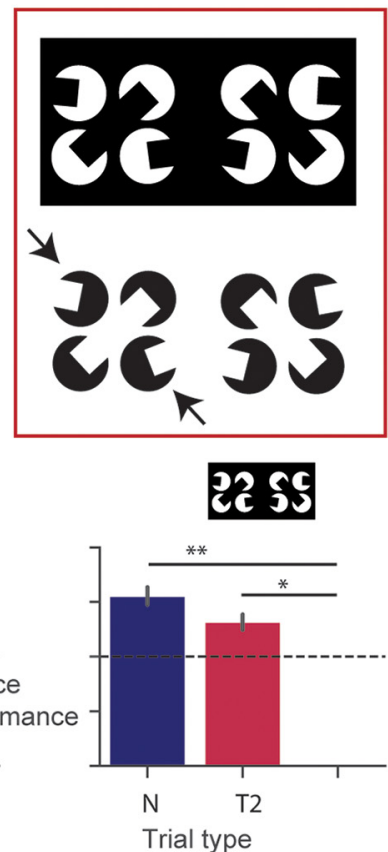

Figure 1. Behavioral correlates of illusory contour perception in mice. $\boldsymbol{A}$, Schematic of an operant conditioning chamber with a touchscreen. $\boldsymbol{B}$, The schedule of the behavioral task. Animals were trained to a touchscreen-based visual discrimination task. It consisted of pre-training (see Materials and Methods), three training stages, and a test stage. During training stages, mice were trained to discriminate between illusory bars of two different orientations $\left(45 \mathrm{vs} 135^{\circ}\right)$. Stages with additional pacmen and color reversal were added to promote animals to learn global rather than local features of the visual stimuli. C, During Test Stage 1, normal trials (with illusory bars) were interleaved with testing trials that contained real bars. Test 2 contained rotated distractor pacmen as an additional control for the local pacmen configuration. $D$, Animals $(n=7)$ were able to learn to discriminate illusory bars of different orientations with a high performance $(75 \%)$. Learning curve by mouse (gray lines) with an overlaid mean (black solid line) shows an increase in performance across several days. $E$, Bar plots show the mean \pm SEM of the performance during normal and testing trials. High performance was observed in normal trials (illusory bars) in both testing stages. During Test Stage 1 trials, animals were able to discriminate real bars with a significantly higher than chance performance despite never being explicitly trained to them. Furthermore, during Test Stage 2 trials, animals were able to discriminate illusory bars despite rotated distractor pacmen. ${ }^{*} p<$ $0.05,{ }^{* *} p<0.01,{ }^{* * *} p<0.001$.

similar visual paradigm as in the prior single-unit and fMRI studies in primates and humans involving the perception of illusory contours in a Kanizsa square (Fig. 2C; Lee and Nguyen, 2001; Maertens et al., 2008). The experiment consisted of several stages: (1) RF mapping, (2) Spike sorting and RF analysis, (3) alignment of RF maps and Kanizsa illusory contours, and (4) recording responses to Kanizsa illusory contours aligned to the RF. We first mapped RF in mouse V1 using locally sparse noise (Zhuang et al., 2017; Fig. 3). After fast spike sorting using Kilosort software ( $\mathrm{Pa}-$ chitariu et al., 2016), we computed spike-triggered average RF maps of the individual units. The Kanizsa square was then aligned to the RF maps so that an illusory contour was on the hotspot of the map but the inducers (pacmen) were outside of it (Fig. 2C).
The radius of inducers was $9.2^{\circ}$ and the side of the square was $44.4^{\circ}$ so that there was a $26^{\circ}$ gap between inducers. Proper positioning of the Kanizsa figure on the monitor screen was chosen to maximize the number of units activated by the subjective contour but not the inducers. Four different types of trials were presented to the animal (Fig. 2D). Each trial started with four circular disks (CIR) followed by one of the four test stimuli: the Kanizsa square containing illusory contours (KIC; red), rotated corners (ROT; green), a filled white square (SQR; dark blue), and a black line square (LINE; purple). Different types of trials were presented in a pseudorandom manner.

We observed that a subset of units in mouse $\mathrm{V} 1$ responds to KIC. They had a much stronger response to KIC compared with 
A

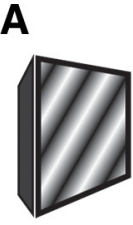

D
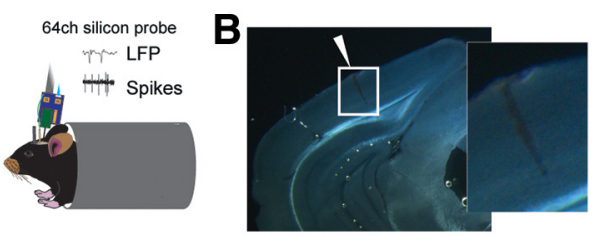

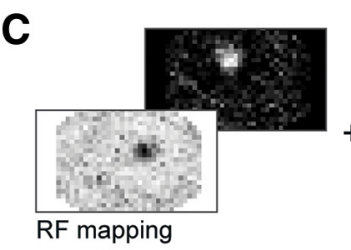

E

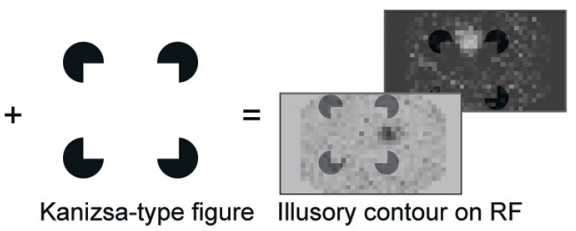

$\mathbf{F}$

$\mathbf{F}$
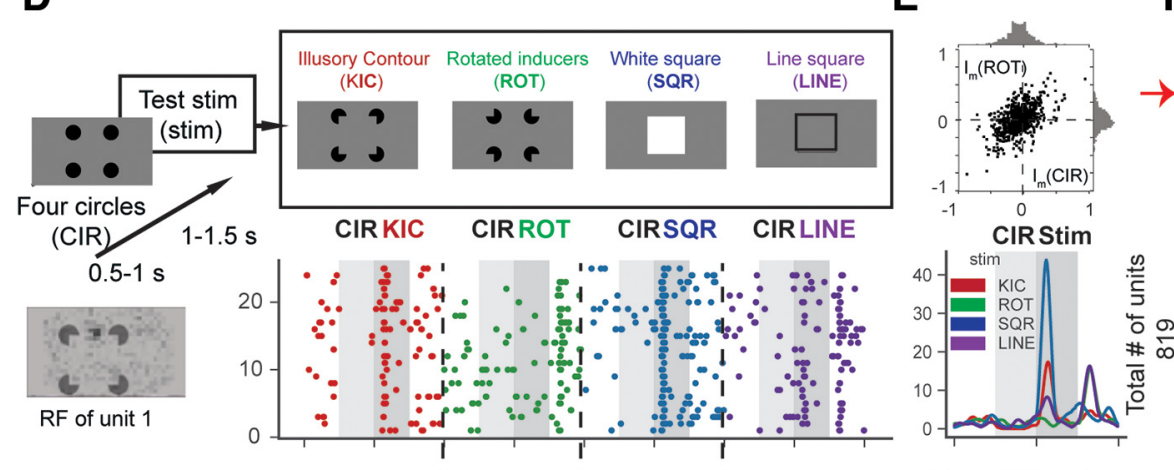

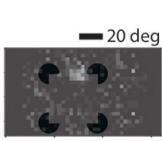

RF of unit 2


Figure 2. Illusory contour responses in a mouse V1. $\boldsymbol{A}$, In vivo extracellular electrophysiology with 64-channel silicon probes in V1 of awake mice. $\boldsymbol{B}$, Histological identification of recording location in V1.C, A Kanizsa figure was presented such that the illusory contour was on the RF of the neuron. Proper positioning of the stimulus on the monitor screen was done by manually aligning the hotspot of the RF maps with an illusory contour of the Kanizsa figure. D, Experimental paradigm: each trial started with four circles (CIR) presented at $0.5-1 \mathrm{~s}$ followed by one of the four test stimuli from 1 to $1.5 \mathrm{~s}$ : Kanizsa illusory contours (red; KIC), rotated corners (green; ROT), white square (blue; SQR), and black line square (purple; LINE). Bottom, Two representative units that responded to the illusory contour but not to rotated corners or circles preceding test stimuli. Raster and PSTH plots show stronger responses to KIC versus ROT or CIR. RF maps of these units are shown on the left and overlaid with Kanizsa illusory contours. $\boldsymbol{E}$, Scatter plot shows the distribution of $I_{\mathrm{m}}(\mathrm{CIR})$ and $I_{\mathrm{m}}(\mathrm{ROT})$ indices across all units. $\boldsymbol{F}$, Heatmap of unit $z$-score responses to four different stimuli $(n=819$ units across 17 recordings from 11 mice). Units were sorted by $F R(K I C)-F R(C I R)$ responses. Note: the red arrow points to a small portion of units at the top of the heatmap that show minimal responses to CIR, but respond to Kanizsa illusory contour presentation.

ROT and CIR (Fig. 2F). To analyze the population-level response to the Kanizsa square, we computed a modulation index by normalizing the responses to the Kanizsa square by those elicited by the circular discs and rotated control: $I_{\mathrm{m}}(\mathrm{CIR})=\mathrm{FR}(\mathrm{KIC})-\mathrm{FR}$ $(\mathrm{CIR}) /[\mathrm{FR}(\mathrm{KIC})+\mathrm{FR}(\mathrm{CIR})]$ and $I_{\mathrm{m}}(\mathrm{ROT})=\mathrm{FR}(\mathrm{KIC})-\mathrm{FR}$ $(\mathrm{ROT}) /[\mathrm{FR}(\mathrm{KIC})+\mathrm{FR}(\mathrm{ROT})]$, where FR refers to the firing rate over $0.05-0.5 \mathrm{~s}$ relative to the stimulus onset, and the $I_{\mathrm{m}^{-}}$ (CIR) and $I_{\mathrm{m}}(\mathrm{ROT})$ indices represent how much KIC was enhanced compared with the CIR and ROT, respectively. For example, positive $I_{\mathrm{m}}(\mathrm{CIR})$ values indicate enhanced responses to the KIC compared with the CIR, whereas negative values mean the opposite. Scatter plot shows the distribution of $I_{\mathrm{m}}(\mathrm{CIR})$ and $I_{\mathrm{m}}(\mathrm{ROT})$ indices for 819 units across 17 recording sessions from 11 mice (Fig. 2E). Distributions of both indices were wide. The top right quadrant represents units with enhanced responses to the KIC compared with CIR and ROT (Fig. 2E). This finding suggests that mouse V1 contains illusory responsive units. Given that we simultaneously recorded many units, we could not adjust the position and orientation of KIC for every single cell. We presented either horizontal or vertical KIC because mouse V1 has a preference for cardinal orientations. Additionally, when considering the relatively large RF size of neurons in mouse V1 and the small proportion of units responsive to KIC that have been found in primate V1 ( 14\%; Lee and Nguyen, 2001), we did not expect to find many illusory responsive units. As can be seen from the heatmap of all recorded units, we did observe a small portion of cells that exhibited a minimal response to inducers but were upregulated by KIC (Fig. 2F, red arrow). Overall, we found single cells that were responsive to illusory contours but not to the circles or the rotated control.

\section{Mouse V1 responds to Kanizsa illusory contours at the population level}

To investigate whether mouse V1 responds to illusory contours at the population level, we averaged unit responses to KIC across the population. We used several criteria to identify illusory modulated units. (1) We identified units that did not have statistically significant responses to four circular discs preceding the KIC stimulus. This was done to ensure that the inducers were outside of the RF. We compared the mean FR between the baseline (0.05$0.5 \mathrm{~s})$ and CIR (0.55-1.0 s) using the Wilcoxon signed rank test. (2) We then used a $I_{\mathrm{m}}$ (CIR) index to identify illusory modulated units, which should have positive indices. In total, 54 units $(6.54 \%)$ satisfied these conditions and were included in further analysis. It is important to note that this is not the proportion of illusory responsive units as the majority of units responsive to KIC were not included because their RF was directly activated by circular discs. The heatmaps and line plots show the neural responses of illusory modulated units to both illusory and real contours (Fig. $4 A, B$ ). Note that the line plots reveal responses to CIR because a small portion of the included units was slightly activated by CIR. We observed that illusory responsive units had significantly stronger population average responses compared with those elicited by the rotated inducers [Fig. $4 C$; mean \pm SEM, 
A

Locally sparse noise (3.84 deg)

B

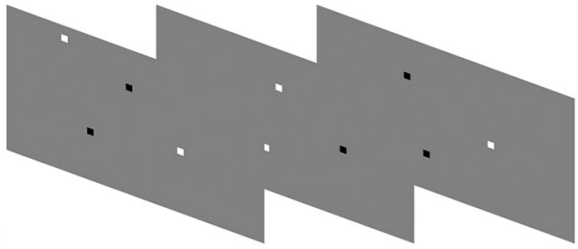

STA RF map

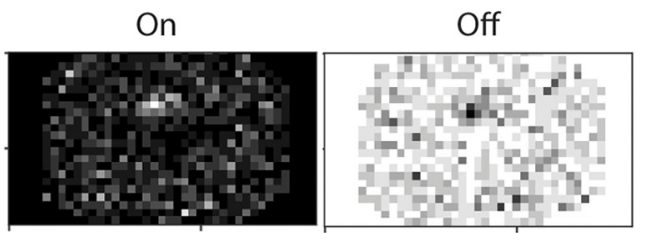

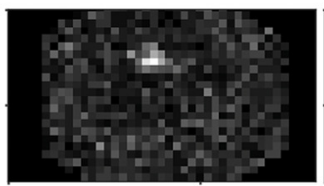

$-15 \mathrm{deg}$
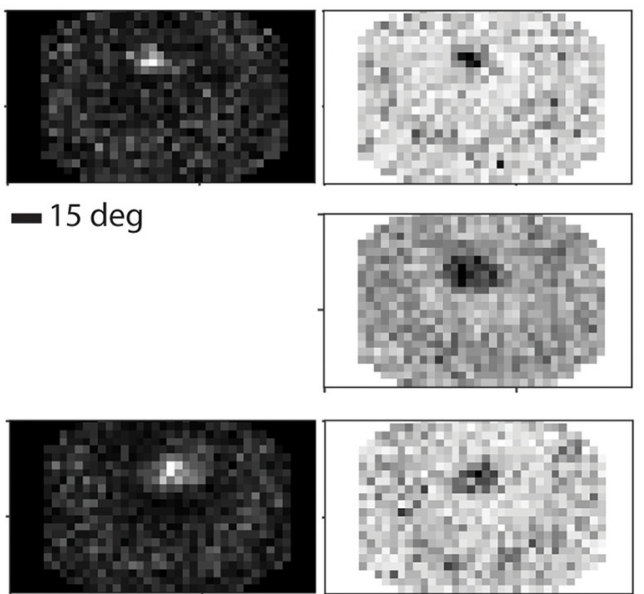

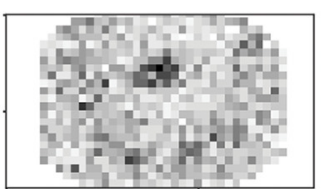

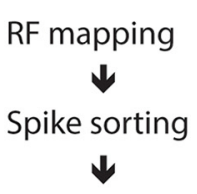

Spike-triggered average (STA)
C

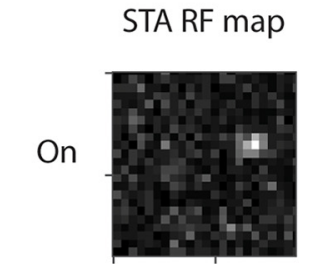

Thesholded 2D Gaussian fit
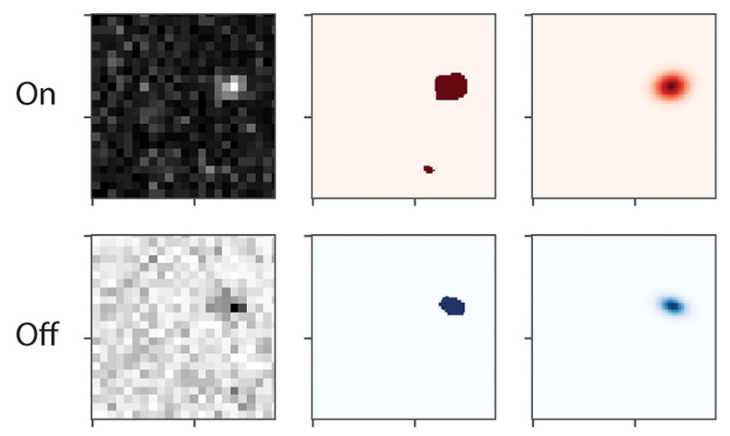

On
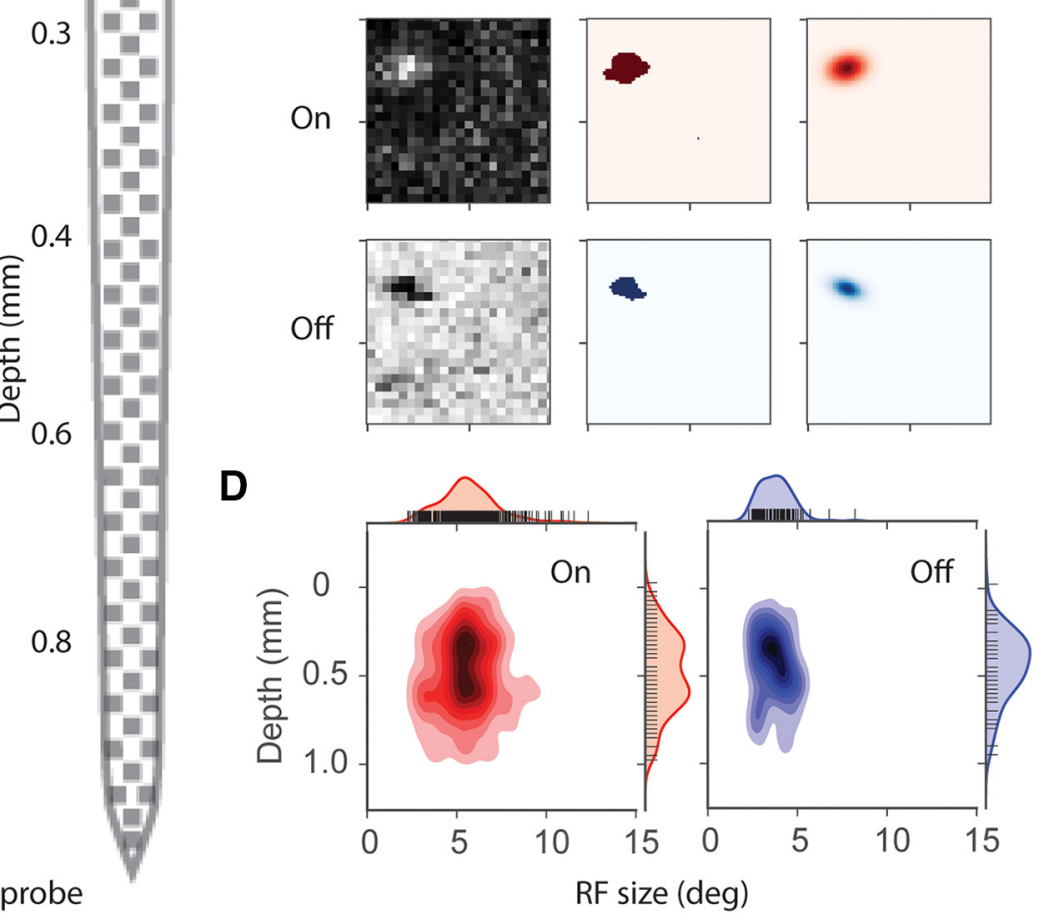

0.8

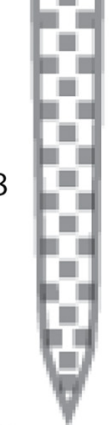

64ch silicon probe

Figure 3. RF mapping and analysis. $A$, Example frames of locally sparse noise stimuli used for RF mapping. After recording, single units were isolated by spike sorting. RF maps were computed by using STA. B, On and Off RF maps across the cortical depth obtained from one recording session. Hotspots represent the putative RF. Diagram of the 64-channel silicon probe is on the right. C, STA RF maps were further analyzed by thresholding outlier pixels and fitting $2 D$ Gaussian. RF size was calculated as the average of the horizontal and vertical half-width at half-maximum of the fitted 2D Gaussian. D, On/Off RF sizes of units were plotted against their putative depth and fitted with KDE. The On RF size was identified for 393 units (48\%) and 0ff for 101 units (12\%).

firing rate: KIC vs ROT (7.03 \pm 0.77 vs $5.67 \pm 0.61 \mathrm{~Hz}, U=871$, $p=0.02)$ vs SQR $(8.37 \pm 0.98 \mathrm{~Hz}, U=1096, p=0.34)$, and vs LINE $(7.01 \pm 0.68 \mathrm{~Hz}, U=1127, p=0.42)$, Mann-Whitney $U$ test, $n=48$ for all comparisons]. The selection criteria were not dependent on the ROT responses as only KIC trials and responses to $\mathrm{CIR}$ were used to identify illusory responsive units. The SQR elicited the strongest responses. Overall, we found significantly stronger responses to KIC versus ROT at the population level.

It has been previously demonstrated in primate V1 that responses to illusory contours are delayed relative to real contours (Lee and Nguyen, 2001). To quantify the response time, we computed the time of the maximal response. Our analysis revealed that KIC responses were significantly delayed relative to SQR but not LINE [Fig. 4D; mean \pm SEM, peak time: KIC vs ROT $(0.17 \pm$ 0.011 vs $0.185 \pm 0.012 \mathrm{~s}, U=372, p=0.09)$, vs $\operatorname{SQR}(0.15 \pm$ $0.006 \mathrm{~s}, U=336, p=0.009)$, and $\operatorname{LINE}(0.17 \pm 0.011 \mathrm{~s}, U=517$, $p=0.44)$, Mann-Whitney $U$ test, $n=33,28,31,32$ respectively]. We also analyzed illusory responses across the cortical depth. We found that the largest mean $I_{\mathrm{m}}$ (ROT) index was observed for the neurons in the superficial layers (Fig. $4 F$; mean \pm SEM. $I_{\mathrm{m}}(\mathrm{ROT})$ : $0.23 \pm 0.079,0.11 \pm 0.028$, and $0.058 \pm 0.024)$. The $I_{\mathrm{m}}(\mathrm{ROT})$ indices were significantly different between neurons within different cortical layers: $\mathrm{L} 2 / 3$ vs L4 $(U=34, p=0.03)$, vs L5/6 ( $U=$ $50, p=0.01)$, L4 vs L5/6 ( $U=118, p=0.09)$, Mann-Whitney $U$ test, $n=9,14$, and 23, respectively.

\section{Illusory contours are orientation-selective in mouse V1}

Illusory contours were shown to be orientation-selective in primates and cats using both Kanizsa type illusions and subjective contours defined by abutting gratings (Peterhans and von der Heydt, 1989; von der Heydt and Peterhans, 1989; Grosof et al., 1993; Sheth et al., 1996). To determine whether mouse V1 KIC responses are orientation selective, we modified our paradigm and presented KIC and other test stimuli of four different orientations (Fig. 5A). We also presented drifting gratings of eight different directions for direction tuning. We ensured that KICs of various orientations overlapped with the RF of the unit, because 
A
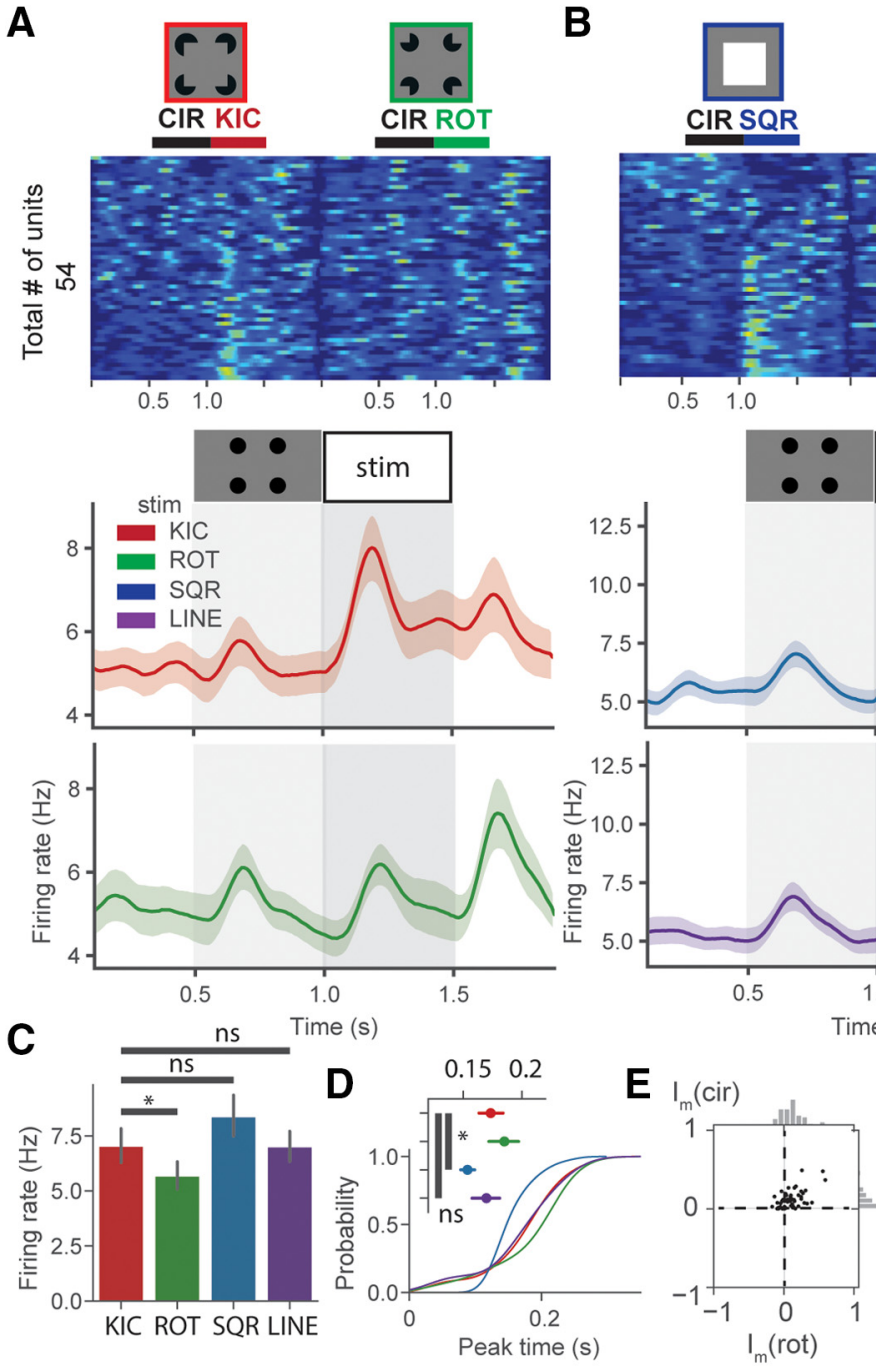

Figure 4. Mouse V1 responds to Kanizsa illusory contours at the population level. $\boldsymbol{A}$, Heatmap of unit $z$-scores in response to illusory contours. Rows represent units; columns, different stimuli. Four circular discs (CIR) were presented at $0.5-1 \mathrm{~s}$ and test stimuli at 1-1.5 s. Bottom, Line plots show the time course of the population mean firing rate to illusory contours, shaded areas represent SEM. B, Heatmap of unit z-scores in response to real contours. Bottom, Line plots represent population mean firing rate to real contours, shaded areas represent SEM. C, Bar plots show the mean \pm SEM of firing rate between 1.05 and $1.5 \mathrm{~s}$ across four different test stimuli. D, Cumulative Distribution Function of peak times (relative to the stimulus onset) of four test stimuli. Inset, Mean \pm SEM peak times across four conditions. $\boldsymbol{E}$, Scatter plot shows the distribution of $I_{\mathrm{m}}(\mathrm{CIR})$ and $I_{\mathrm{m}}(\mathrm{ROT})$ indices of units responsive to illusory contours. $\boldsymbol{F}$, Distribution of $I_{\mathrm{m}}(\mathrm{ROT})$ indices in L2/3, L4, and L5/6. $p<0.05$ was considered significant. ${ }^{*} p<0.05$. ns $=$ not significant.

the absence of responses to KIC of a particular orientation may be due to the RF being outside of the visual stimulation. To address this potential issue, we determined whether units still responded to real contours while not responding to KIC. We found that a subset of units responded to KIC of only one orientation but had responses to real contours (SQR or LINE) of various orientations (Fig. 5B). We manually identified units that showed minimal responses to CIR in at least two conditions (KIC orientations). In total, we identified 15 units from 6 recording sessions from 3 animals. However, a subset of the units had observable responses to CIR. To account for that, we used $I_{\mathrm{m}}$ (CIR) indices to construct a putative population KIC tuning curve. One of the limitations of this experiment is the use of static KIC stimuli because the response magnitude will depend on the relative position of the contour relative to the RF of each neuron. We also analyzed the direction tuning of these units using drifting grating stimuli (Fig. $5 A$ ). To generate population-tuning curves for gratings and illu-

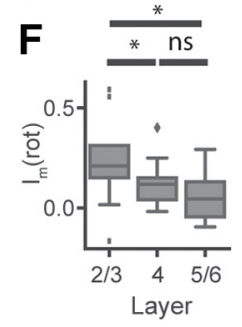

sory contours, we first identified the orientation that induced the maximal response. Responses were then rescaled to $0-1$ and plotted relative to the preferred orientation. We then set the preferred orientation as $0^{\circ}$, and shifted all the other orientations accordingly. For example if the preferred orientation of the unit was $45^{\circ}$, then $45^{\circ}$ was subtracted from all the orientations. The unit responses were then plotted against $-45,0,45,90^{\circ}$ instead of $0,45,90$, and $135^{\circ}$. The response at $0^{\circ}$ would be equal to 1 as it was the maximal response. Given the clear peak in the population tuning curve obtained with drifting gratings, these units were determined to be orientation-selective [Fig. $5 F$; at least one group median is different from others $(H=29.4, p=1.87 \mathrm{E}-5)$, $n=14$, Kruskal-Wallis test]. The putative population KIC tuning curve had a similar shape and at least one group was significantly different from others (Fig. $5 D$; $H=16.6, p=0.005 ; n=15$, KruskalWallis test). We also performed the same analysis for ROT, SQR, and LINE. $I_{\mathrm{m}}$ (CIR) was computed separately for each stimulus by normalizing their responses by CIR similarly as for KIC. We did not observe significant orientation tuning for rotated inducers, which further supports that putative KIC tuning was due to the illusory contours (Fig. $5 D ; H=9.84, p=$ $0.07 ; n=14$, Kruskal-Wallis test). Interestingly, we also observed significant orientation tuning for LINE (Fig. $5 E ; H=$ 11.5, $p=0.04 ; n=14$, Kruskal-Wallis test) but not for $\mathrm{SQR}$ responses (Fig. $5 E$; $H=7.5, p=0.18 ; n=15$, Kruskal-Wallis test). We also analyzed the distribution of preferred orientations obtained with drifting gratings versus KIC (Fig. 5G) and found a good correspondence between them. Together, our results suggest that illusory responses are orientationselective in mouse V1.

\section{Top-down feedback from LM modulates illusory contour responses in $\mathrm{V} 1$}

To test the hypothesis that top-down feedback is important for the responses to illusory contours in V1, we used electrophysiological recordings in V1 combined with optogenetic inhibition of the LM visual area. We injected adeno-associated virus (AAV) expressing ArchT, a light-sensitive outward proton pump (Han et al., 2011), and GFP into LM, which is analogous to primate V2 and is known to send feedback projections to $\mathrm{V} 1$ and is the most interconnected area with V1 (Fig. 6A,B; Wang and Burkhalter, 2007; Pan et al., 2012). Three weeks after injection, we performed in vivo electrophysiology with optogenetics. We first verified that optogenetic inactivation of LM suppressed responses to grating stimuli in LM but only modulated responses in V1. We recorded neural activity in V1 while an optical fiber was positioned above LM. Optogenetic trials were in- 
A

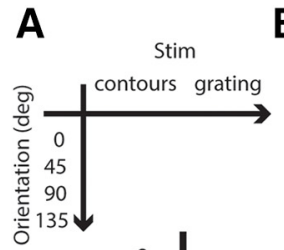

B stim

KIC SQR grating ROT LINE

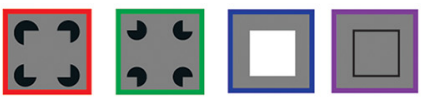

CIR KIC CIRROT CIRSQR CIRLINE

0

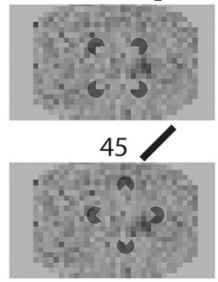

$90-$

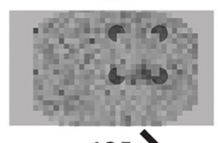

135

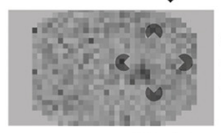

- $16 \mathrm{deg}$
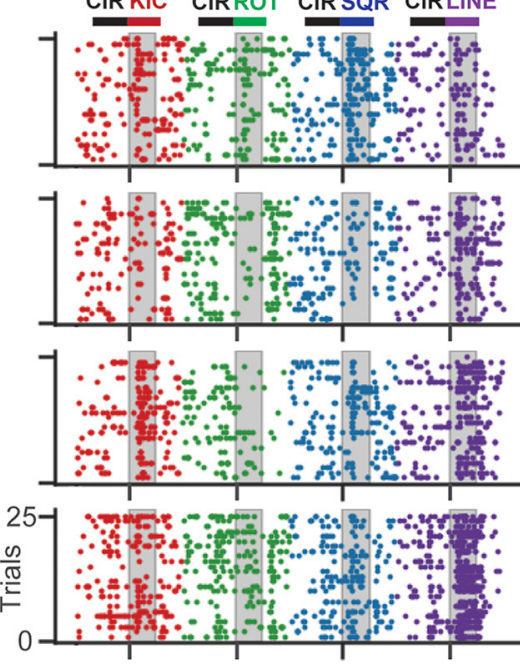

C
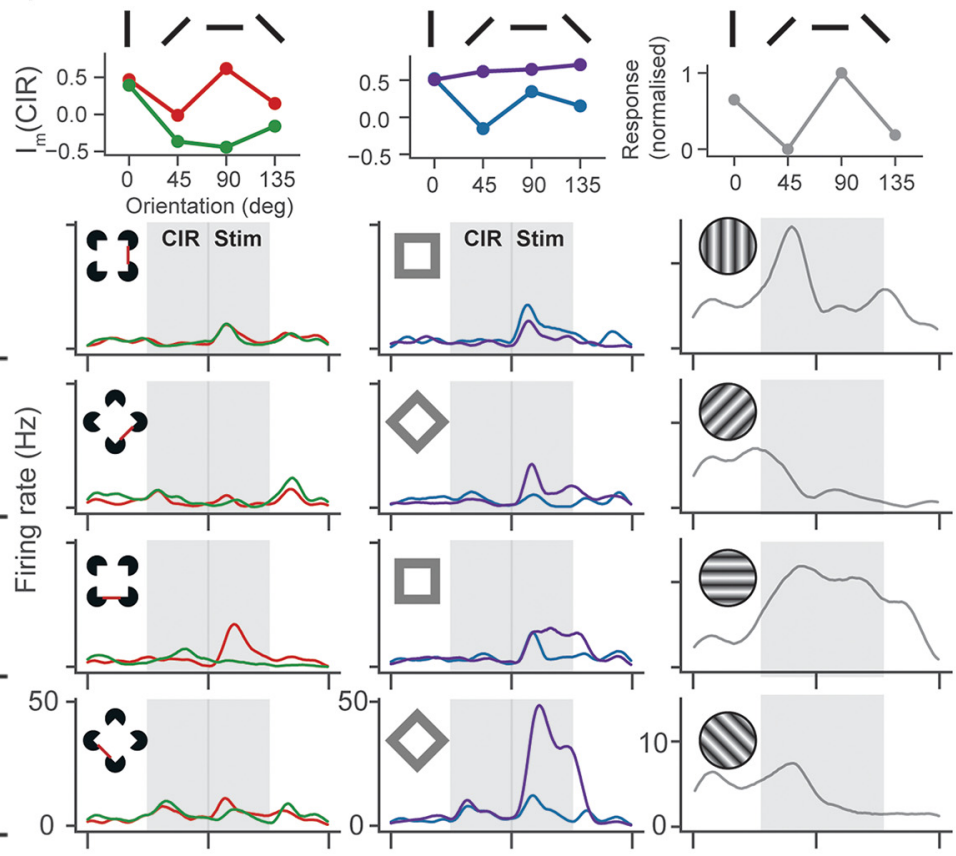

$\begin{array}{llllllll}0 & 1.0 & 0 & 1.0 & 0 & 1.0 & 0 & 1.0\end{array}$

0

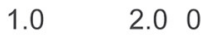

$1.0 \quad 2.0 \quad 0$

$0 \quad 0.5$

0.5

D

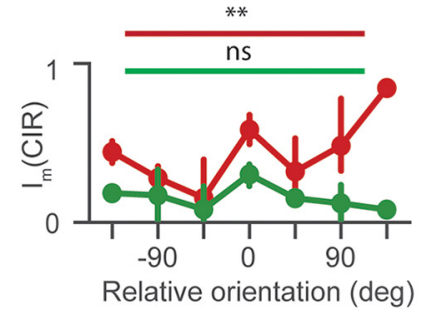

E

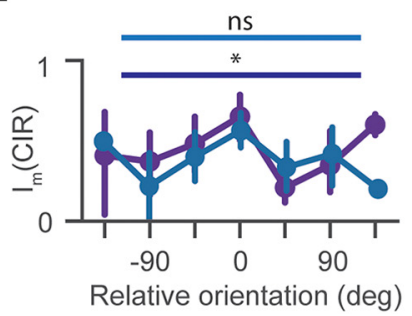

$\mathbf{F}$

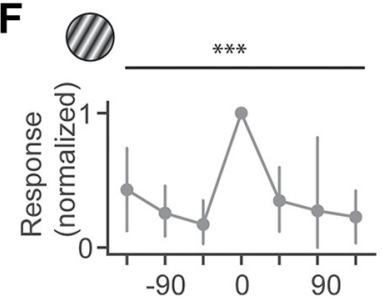

Relative orientation (deg)
G

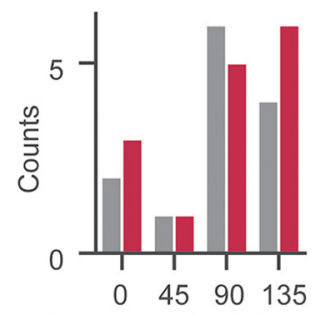

Preferred orientation (deg)

Figure 5. Illusory contours are orientation selective in mouse V1. A, Arrows point to different stimuli versus orientations. RF map below is overlaid with KIC and shows the orientation of illusory contours presented to the mouse. $\boldsymbol{B}$, Raster plots for the representative units show responses in 16 different conditions (4 test stimuli vs 4 orientations). Columns represent four different stimuli, whereas rows show the responses of real and illusory contours of different orientations. $\boldsymbol{C}$, Line plot shows the mean firing rate in response to four test stimuli and grating (rightmost) across four different orientations. Putative tuning plots are shown at the top. $\boldsymbol{D}$, Putative population $\mathrm{KIC}$ and ROT tuning curve of $I_{\mathrm{m}}(\mathrm{CIR})$ responses relative to maximum response orientation: We used positive $I_{m}(C I R)$ values to only include units with minimal CIR responses. $\boldsymbol{E}$, Same as in $\boldsymbol{D}$ but for SQR and LINE. Note: $I_{\mathrm{m}}(\mathrm{CIR})$ represents normalized to CIR responses for different test stimuli. $\boldsymbol{F}$, Population tuning curve obtained with drifting grating stimuli. Responses were rescaled to $0-1$ before averaging. Responses were plotted relative to the preferred orientation of the unit. $\mathbf{G}$, Bar plot shows the counts of units preferring different orientations mapped with either drifting gratings or KIC. $p<0.05$ was considered significant. ${ }^{*} p<0.05,{ }^{* *} p<0.01,{ }^{* * *} p<0.001$. ns $=$ not significant.

terleaved with regular trials (Figs. 2, 4). First, we calculated a KIC optogenetic modulation index for each unit: $\mathrm{I}_{\mathrm{m}}$ (laser) $=$ $[\mathrm{FR}(\mathrm{KIC})-\mathrm{FR}(\mathrm{KIC}+$ laser $)] /[\mathrm{FR}(\mathrm{KIC})+\mathrm{FR}(\mathrm{KIC}+$ laser $)]$. This index represents the KIC response normalized by the KIC response during LM inhibition. Positive $I_{\mathrm{m}}$ (laser) values indicate a decrease in response to KIC during LM inhibition compared with the absence of LM inhibition, whereas negative values indicate the opposite. Scatter plots show the distribution of $I_{\mathrm{m}}$ (laser) and $I_{\mathrm{m}}$ (ROT) indices (Fig. $6 C$ ). The majority of illusory modulated units were in the top right quadrant of the scatter plot, suggesting that optogenetic silencing of LM decreased KIC responses. Three representative units show responses to KIC with (solid colors) and without (light colors) LM inhibition (Fig. 6D). We saw a clear decrease in KIC but not ROT responses during LM inhibition. Heatmaps and line plots show the population responses during the optogenetic experiment (Fig. $6 E, F)$. We found that the mean KIC but not ROT responses significantly decreased during optogenetic inhibition of LM [Fig. $6 G$; mean \pm SEM of firing rate: KIC vs KIC + laser $(6.69 \pm 0.72$ vs $5.72 \pm$ $0.52 \mathrm{~Hz}, \mathrm{~W}=306, p=0.0004), \mathrm{ROT}$ vs ROT + laser $(5.43 \pm 0.58 \mathrm{vs}$
$5.65 \pm 0.61 \mathrm{~Hz}, \mathrm{~W}=572, p=0.28)$, Wilcoxon signed rank test, $n=$ 52 for both comparisons]. Scatter plots show the single-unit responses during LM inhibition. Together, these results suggest that top-down feedback controls Kanizsa illusory contour responses in mouse V1.

Optogenetic stimulation did not affect responses to SQR and LINE in illusory responsive units (Fig. 7). This suggests that the downregulation of KIC responses cannot be attributed to direct optogenetic inhibition of V1. Interestingly, laser stimulation did modulate responses to SQR but not LINE in units that had significant responses to CIR (Fig. 8). These results suggest that optogenetic silencing of LM might differentially modulate responses of different neuronal groups in V1.

\section{Discussion}

Our study suggests that one of the ways top-down feedback influences sensory processing in V1 is by supplying the missing information. We first established that mice can distinguish Kanizsa illusory contours using behavior. Second, we demon- 
A

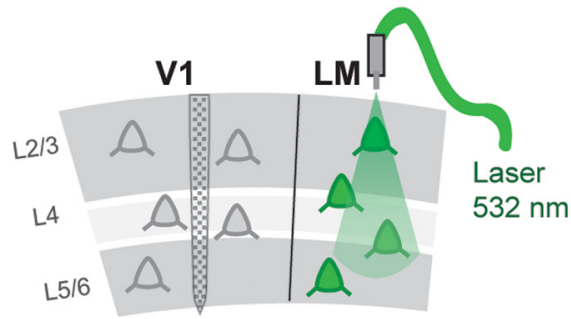

D
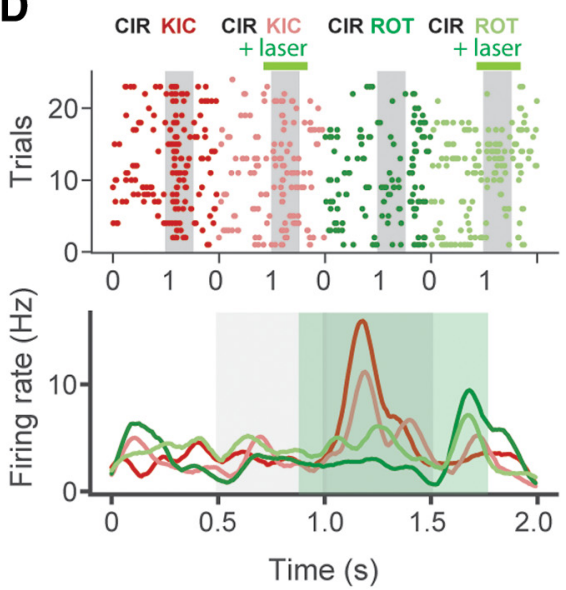

E

言
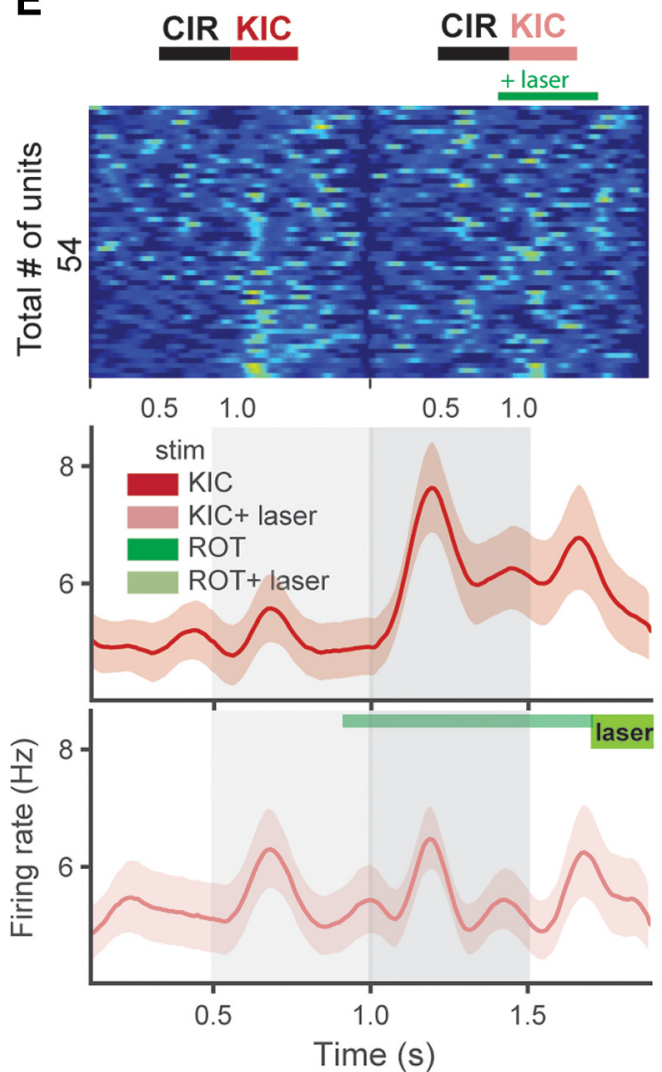

B

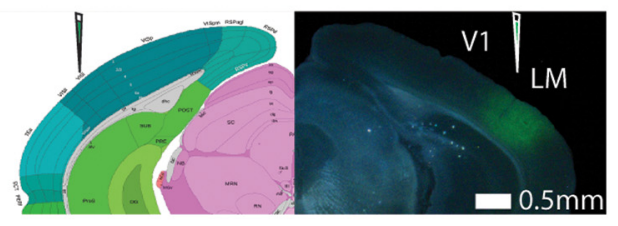

C

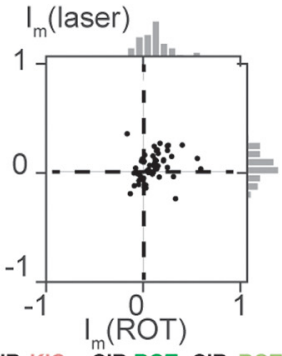

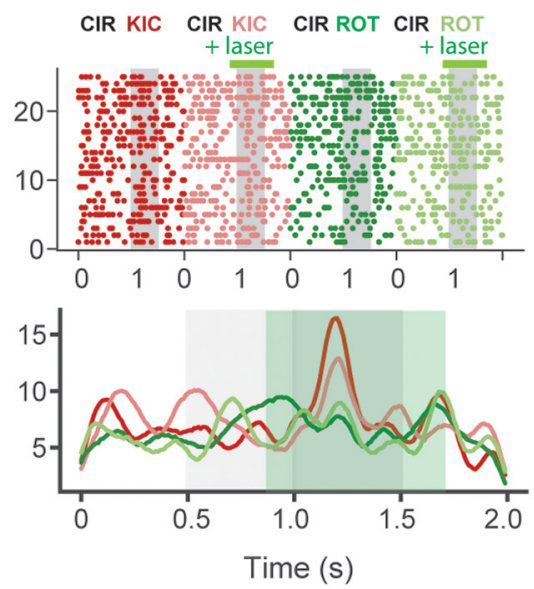

F
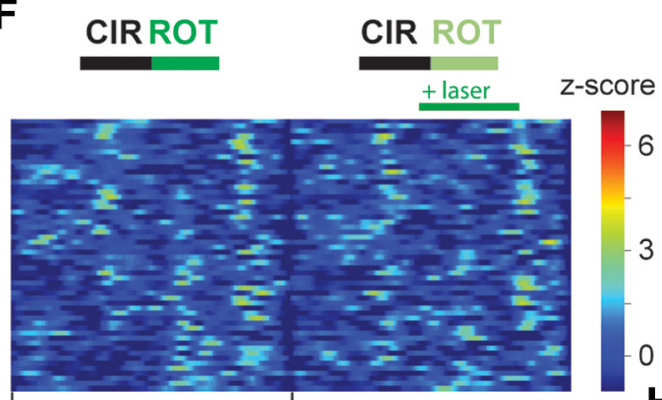

$0.5 \quad 1.0$
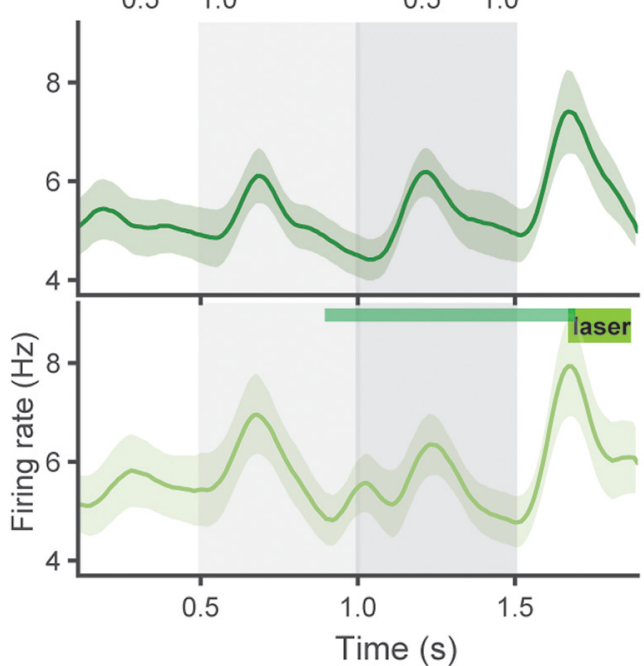

0
CIR KIC CIR KIC CIR ROT CIR ROT
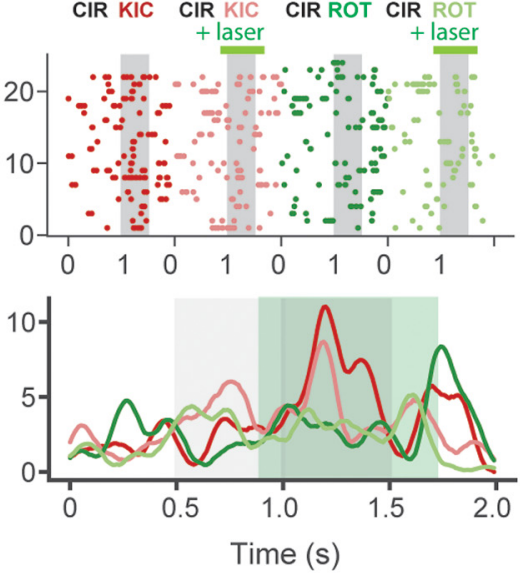

G
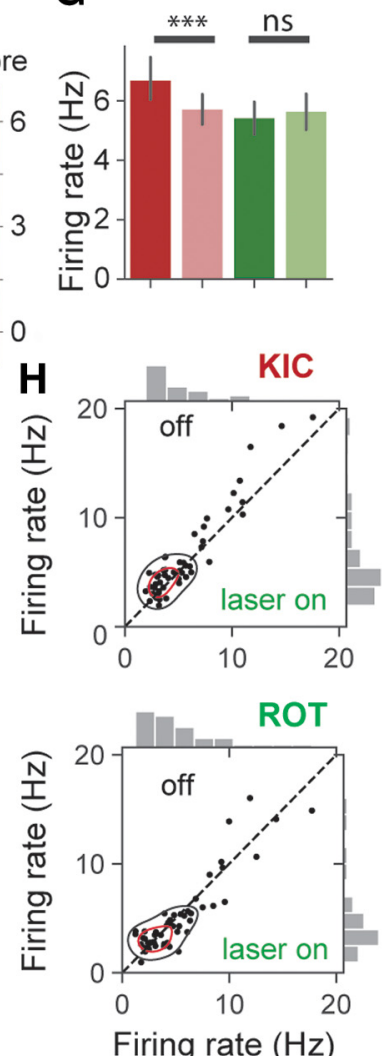

Firing rate $(\mathrm{Hz})$

Figure 6. Top-down feedback from LM controls illusory responses in mouse V1.A, ArchT was injected into the LM area and electrophysiological recordings were performed in V1. An optical fiber was positioned above $L M$ to inactivate the region with green light $(532 \mathrm{~nm}) . \boldsymbol{B}$, Injection location and viral spread were verified using histology. $C$, The scatter plot shows the distribution of $I_{\mathrm{m}}(\mathrm{ROT})$ and $I_{m}$ (laser) indices. $\boldsymbol{D}$, Representative raster plots along with line plots show illusory responses with (solid colors) and without (light colors) LM inactivation. $\boldsymbol{E}$, Heatmap of unitz-scores in response to KIC with (left) and without (right) LM inactivation. Bottom: Responses to the ROT stimulus. F, Line plots show the mean firing rate of units in the heatmap. Responses to KIC (red) and ROT (green) stimuli with (solid colors) and without (light colors) LM inactivation. Circular discs (CIR) were presented at $0.5-1 \mathrm{~s}$ and test stimuli at 1-1.5 s. Laser light was applied from 0.9 to $1.7 \mathrm{~s}$ as shown with the green rectangle at the bottom of the line plots. $G$, Bar plots show the mean \pm SEM of firing rate between 1.05 and 1.5 s across four different conditions. $\boldsymbol{H}$, Scatter plots of single-unit responses to KIC (top) and ROT (bottom) during optogenetic inactivation of LM ( $x$-axis) and without it $\left(y\right.$-axis). $p<0.05$ was considered significant. ${ }^{* * *} p<0.001$. ns $=$ not significant. 
strated that mouse $\mathrm{V} 1$ responds to illusory contours. We then showed that the neurons responsive to illusory contours are orientation-selective. Finally, we provided neurophysiological evidence that top-down feedback controls illusory contour responses in mouse V1.

Our behavioral data are consistent with the previous work and strongly suggest that mice can perceive illusory contours (Okuyama-Uchimura and Komai, 2016). Our findings from the transfer stages with luminance-defined bars provide compelling evidence that animals were relying on the global features of the visual stimuli to perform the tasks. Total luminance was different between the illusory bars images containing extra pacmen compared with the images with real bars. Because the mice had not seen the real bars during the training stages, the only strategy to discriminate between the real bars would require learning to discriminate between the illusory bars during training. Not all the mice were able to pass the training stages possibly because of the different strategies they adopt to perform the task. Early stages of training do not require mice to rely on global features to pass the stage. However, mice would not be able to pass a color inversion stage if they relied on a local luminance distribution in the visual stimuli. Consistent with this explanation, all the animals that did not reach the testing stages failed at the color inversion stage. Although this finding does not mean the animals are unable to perceive illusory bars, it suggests that they did not rely on the global features during training.

Our neural data are consistent with the previous single-unit studies describing illusory contour responses in primate V1 (Lee and Nguyen, 2001) and figureground modulation in mouse V1 (Schnabel et al., 2018). Consistent with the previous reports of delayed responses to illusory contours compared with real contours, we also observed a $30 \mathrm{~ms}$ delay in responses to illusory compared with real contours. Furthermore, we found stronger illusory modulation in the superficial layers, the target of the feedback axonal projections from the secondary visual area LM (Wang and Burkhalter, 2007; Pan et al., 2012). This observation is also consistent with prior primate work (Lee and Nguyen, 2001) and a more recent human fMRI study (Lawrence et al., 2019) investigating laminar modulation by top-down feedback. However, another human fMRI study demonstrated stronger deep layer activation during Kanizsa presentation (Kok et al., 2016). This discrepancy between the mouse and human data could potentially be explained by the differences in the anatomy of mice and humans or by the experimental paradigms. In support of the notion that mice can distinguish illusory contours is the fact that
A
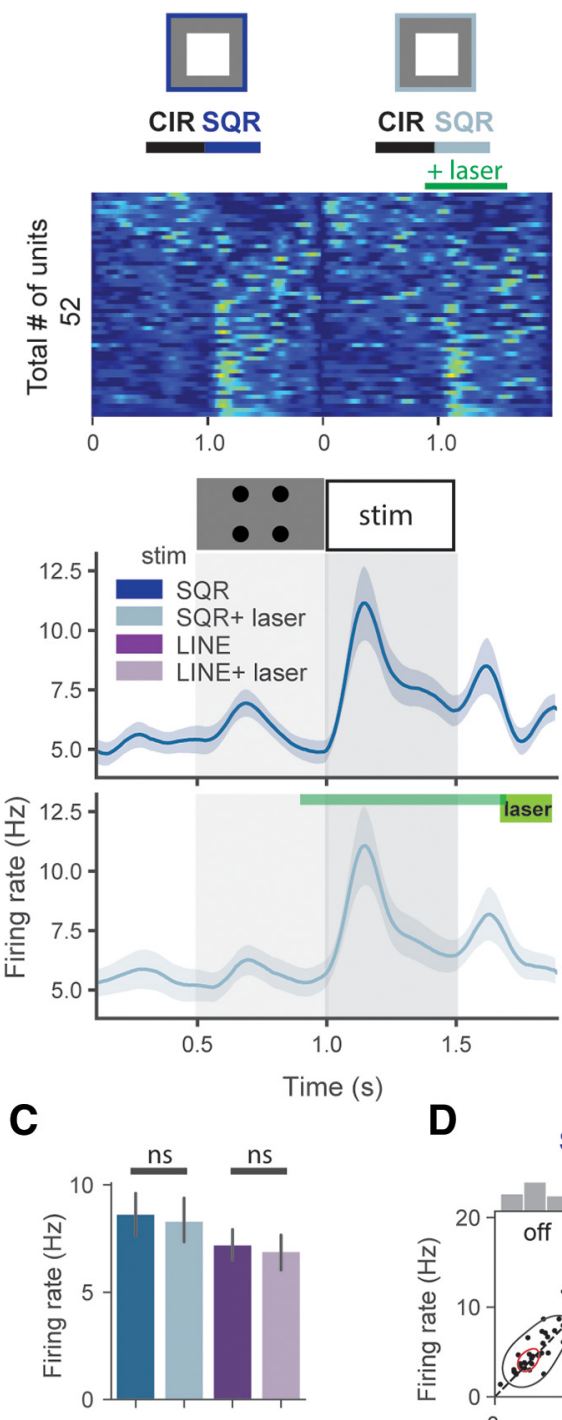

B
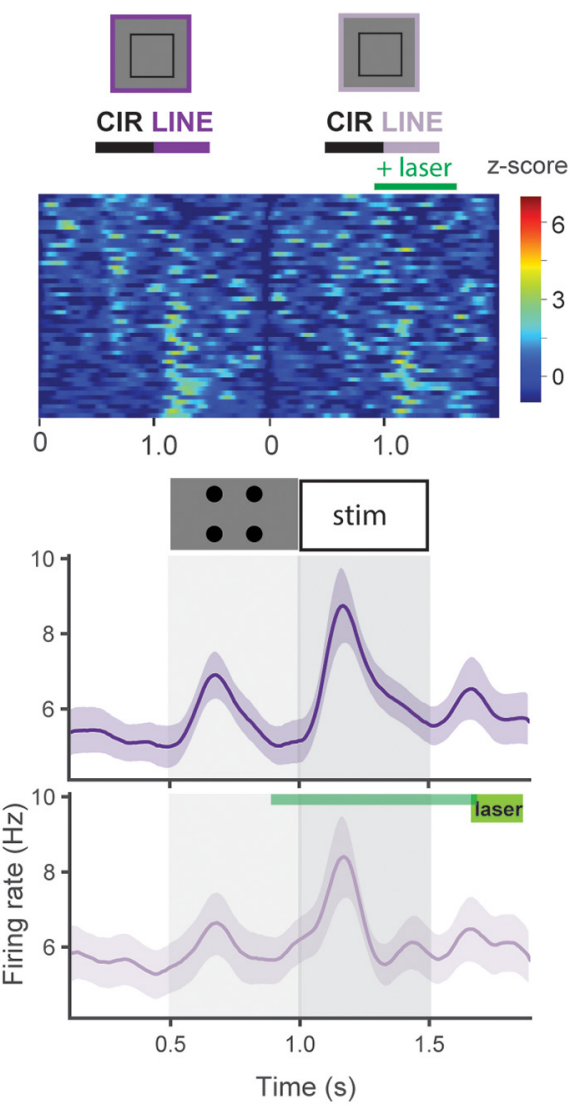

SQR

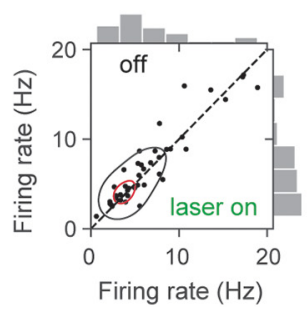

Figure 7. Inactivation of top-down feedback does not affect real contour responses in illusory modulated units. $\boldsymbol{A}$, Heatmaps of unit z-scores to real contours. Each column represents responses to different stimuli. Responses to SQR (blue) and LINE (purple) with (light colors) and without (dark colors) green $(532 \mathrm{~nm}$ ) laser light to optogenetically inhibit LM. Circular discs (CIR) were presented at $0.5-1 \mathrm{~s}$ and test stimuli at 1-1.5 s. Laser stimulation was applied from 0.9 to $1.7 \mathrm{~s}$. $\boldsymbol{B}$, Line plots represent the time course of mean firing rate to the SQR (top) and LINE (bottom). C, Bar plots shows the mean \pm SEM of firing rate between 1.05 and 1.5 s across four different conditions: SQR vs SQR + laser ( $8.64 \pm 1.00$ vs $8.32 \pm 1.03 \mathrm{~Hz}, \mathrm{~W}=420, p=0.18)$, LINE vs LINE + laser $(7.21 \pm 0.69$ vs $6.90 \pm 0.79 \mathrm{~Hz}, W=382, p=0.09)$, Wilcoxon signed rank test; $n=46$ for both comparisons. $D$, Scatter plots show single-unit $z$-score responses with ( $x$-axis) and without ( $y$-axis) LM inactivation. Dashed line represents a unity line. $p<0.05$ was considered significant. ns $=$ not significant.

the KIC responses in the mouse V1 are orientation-selective. Orientation selectivity of Kanizsa illusory contours has been demonstrated in primates with high degree of correlation between the tuning curves obtained with real and illusory contours (Peterhans and von der Heydt, 1989; von der Heydt and Peterhans, 1989; Sheth et al., 1996).

Our optogenetic experiments suggest that top-down feedback from LM modulates illusory contour responses in V1. Our data are consistent with the prior primate lesion study and the recurrent processing theories of illusory contours perception (De Weerd et al., 1996; Wyatte et al., 2014). One of the challenges of our study was the proximity of LM to V1. However, our histology 
A Units with a significant $\mathrm{CIR}$ responses
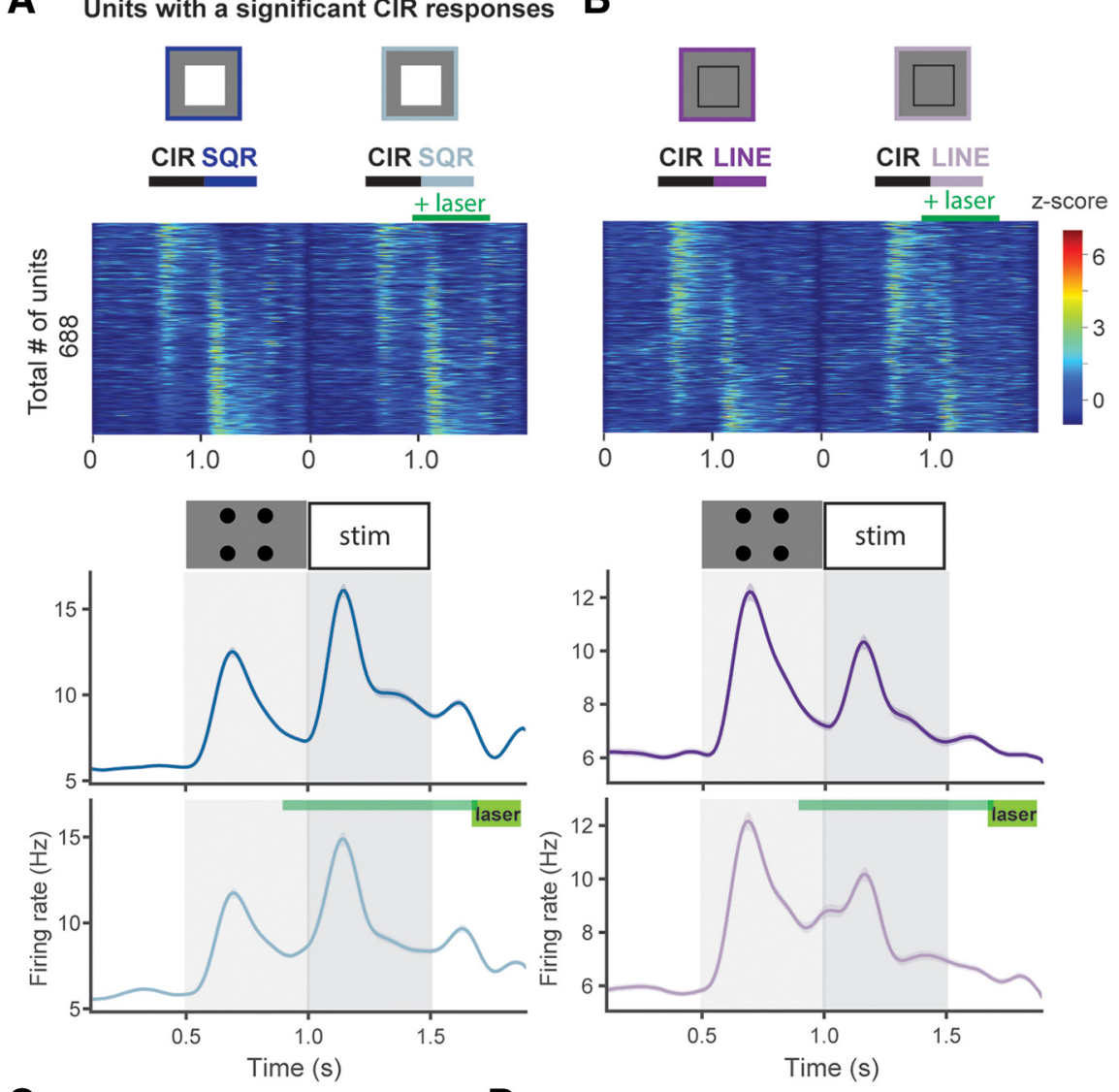

C

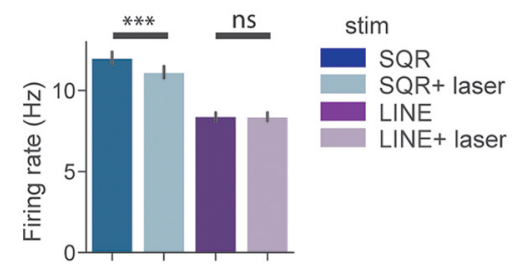

can be explained by the extensive innervation of V1 by LM and is consistent with the study reporting that top-down feedback modulates feature tuning in V1 (Huh et al., 2018). It is unlikely to be because of the direct V1 inhibition because LINE responses were not affected. It is not clear why SQR responses were not modulated in KIC responsive neurons. This finding could be potentially explained by the distinct LM connectivity and modulation patterns of different neuronal subpopulations in V1.

What is the mechanism of the illusory contour perception? Recurrent processing is required for illusory contour responses (Mendola et al., 1999; Lee and Nguyen, 2001; Pan et al., 2012). Early lesion studies in primates and more recent studies have directly shown the importance of recurrent processing for perception of visual illusions (De Weerd et al., 1996; Luo et al., 2019). The interplay between bottom-up and top-down signals may play a crucial role in illusory shape perception. One plausible mechanism for the illusory responses in early visual cortex might be the top-down feedback modulating effective local connectivity in V1. It has been recently shown that the top-down feedback from V4 to V1 can control the effective connectivity of lateral connections in V1 during a contour detection task in monkeys (Liang et al., 2017). Contour completion that occurs in illusory shapes might be mediated via the above mechanism; similarly oriented edges along one axis would activate units in the higher visual areas that, in turn, could facilitate contour integration by modulating lateral connectivity in V1. Such a mechanism would be robust even in the presence of breaks in the contour continuity.

Recent theories and computational recurrent network models were proposed to explain the illusory contour responses (Peterhans and von der Heydt, 1989; Lee, 2003). One of the proposed models suggests that neurons in V2 might pool orientation selective feedforward inputs

and neural data suggest that optogenetic activation was specific to LM. To decrease the likelihood of direct inhibition of V1, we injected a small amount of the construct $(50 \mathrm{nl})$ to minimize the spread of the virus, which resulted in localized injections. Second, optical fiber was painted with the black ink and couplers covered with the foil to restrict light illumination only to LM. Finally, neural responses by illusory modulated neurons to SQR and LINE stimuli were not affected by LM inhibition. Together, these observations suggest that the downregulation of illusory contour responses in V1 was mainly because of LM suppression.

We observed that optogenetic suppression of LM resulted in the downregulation of SQR, but not LINE responses considering all recorded neurons but KIC modulated ones. This observation from a set of V1 units that were activated by local inducers. Then, activated V2 cells can provide spatially nonspecific but featurespecific feedback to those neurons, instructing them about the global context. Feedback signal will thus facilitate contour completion by directly upregulating its V1 targets or modulating them indirectly via recurrent activity (Mignard and Malpeli, 1991). Interestingly, a recent deep convolutional recurrent neural network, PredNet, which represents an implementation of the predictive coding theory, recapitulated dynamics of illusory contour responses as observed in the primate single-unit study (Lotter et al., 2018) and in our experiments. Predictive coding postulates that top-down feedback from the higher visual areas 
conveys expectations about sensory information, while the bottom-up feedforward pathways carry the sensory information. This sensory information is then subtracted from the predictions generated at the higher computational level. According to this framework, the predictions about the illusory shape are sent to V1. Consistent with this theory, human fMRI study showed that V1 responses to the Kanizsa illusion depended on whether the input information was predicted by the bottom-up signals. Authors separately analyzed responses of the regions that corresponded to the illusory shape and inducers that formed the illusion. They compared the responses to the condition with rotated pacmen that did not produce the illusion and discovered the upregulated responses to the illusory shape and downregulated responses to the inducers. The authors concluded that the effect could be attributed to the predictions about the stimuli: pacmen induced the bottom-up signals that were predicted, whereas the illusory shape did not. The responses to the unpredicted visual inputs such as the illusory shape, were upregulated, whereas the responses to the inducers that formed the illusion were suppressed (Kok and de Lange, 2014). We did not find similar effects in our study, which might be because of the differences in approach and/or species we used.

In conclusion, our findings suggest that top-down feedback is important for the generation of neural correlates of Kanizsa illusory perception. This feedback is feature-specific, because of the observed orientation selectivity of KIC in mouse V1. Future mechanistic studies of illusory perception in mice, a genetically tractable model system, will greatly facilitate our understanding of perceptual inference in the visual system and help us elucidate the neural computations underlying it.

\section{References}

Bar M, Kassam KS, Ghuman AS, Boshyan J, Schmidt AM, Dale AM, Hämäläinen MS, Marinkovic K, Schacter DL, Rosen BR, Halgren E (2006) Top-down facilitation of visual recognition. Proc Natl Acad Sci U S A 103:449-454.

De Weerd P, Desimone R, Ungerleider LG (1996) Cue-dependent deficits in grating orientation discrimination after V4 lesions in macaques. Vis Neurosci 13:529-538.

Gilbert CD, Li W (2013) Top-down influences on visual processing. Nat Rev Neurosci 14:350-363.

Grosof DH, Shapley RM, Hawken MJ (1993) Macaque VI neurons can signal "illusory" contours. Nature 365:550-552.

Han X, Chow BY, Zhou H, Klapoetke NC, Chuong A, Rajimehr R, Yang A, Baratta MV, Winkle J, Desimone R, Boyden ES (2011) A high-light sensitivity optical neural silencer: development and application to optogenetic control of non-human primate cortex. Front Syst Neurosci 5:18.

Horner AE, Heath CJ, Hvoslef-Eide M, Kent BA, Kim CH, Nilsson SR, Alsiö J, Oomen CA, Holmes A, Saksida LM, Bussey TJ (2013) The touchscreen operant platform for testing learning and memory in rats and mice. Nat Protoc 8:1961-1984.

Huh CY, Peach JP, Bennett C, Vega RM, Hestrin S (2018) Feature-specific organization of feedback pathways in mouse visual cortex. Curr Biol 28:114-120.e115.

Kanizsa G (1976) Subjective contours. Sci Am 234:48-52.

Kanizsa G, Renzi P, Conte S, Compostela C, Guerani L (1993) Amodal completion in mouse vision. Perception 22:713-721.

Kissinger ST, Pak A, Tang Y, Masmanidis SC, Chubykin AA (2018) Oscillatory encoding of visual stimulus familiarity. J Neurosci 38:6223-6240.

Kok P, de Lange FP (2014) Shape perception simultaneously up- and downregulates neural activity in the primary visual cortex. Curr Biol 24:1531-1535.

Kok P, Bains LJ, van Mourik T, Norris DG, de Lange FP (2016) Selective activation of the deep layers of the human primary visual cortex by topdown feedback. Curr Biol 26:371-376.

Lawrence SJ, Norris DG, de Lange FP (2019) Dissociable laminar profiles of concurrent bottom-up and top-down modulation in the human visual cortex. eLife 8:e44422.
Lee TS (2003) Computations in the early visual cortex. J Physiol Paris 97:121-139.

Lee TS, Nguyen M (2001) Dynamics of subjective contour formation in the early visual cortex. Proc Natl Acad Sci U S A 98:1907-1911.

Liang H, Gong X, Chen M, Yan Y, Li W, Gilbert CD (2017) Interactions between feedback and lateral connections in the primary visual cortex. Proc Natl Acad Sci U S A 114:8637-8642.

Li W, Piëch V, Gilbert CD (2004) Perceptual learning and top-down influences in primary visual cortex. Nat Neurosci 7:651-657.

Lotter W, Kreiman G, Cox D (2018) A neural network trained to predict future video frames mimics critical properties of biological neuronal responses and perception. arXiv:180510734.

Luo J, He K, Andolina IM, Li X, Yin J, Chen Z, Gu Y, Wang W (2019) Going with the flow: the neural mechanisms underlying illusions of complexflow motion. J Neurosci 39:2664-2685.

Maertens M, Pollmann S, Hanke M, Mildner T, Möller H (2008) Retinotopic activation in response to subjective contours in primary visual cortex. Front Hum Neurosci 2:2.

Mendola JD, Dale AM, Fischl B, Liu AK, Tootell RB (1999) The representation of illusory and real contours in human cortical visual areas revealed by functional magnetic resonance imaging. J Neurosci 19:8560-8572.

Mignard M, Malpeli JG (1991) Paths of information flow through visual cortex. Science 251:1249-1251.

Murray MM, Wylie GR, Higgins BA, Javitt DC, Schroeder CE, Foxe JJ (2002) The spatiotemporal dynamics of illusory contour processing: combined high-density electrical mapping, source analysis, and functional magnetic resonance imaging. J Neurosci 22:5055-5073.

Nieder A (2002) Seeing more than meets the eye: processing of illusory contours in animals. J Comp Physiol A Neuroethol Sens Neural Behav Physiol 188:249-260.

Niell CM, Stryker MP (2008) Highly selective receptive fields in mouse visual cortex. J Neurosci 28:7520-7536.

Okuyama-Uchimura F, Komai S (2016) Mouse ability to perceive subjective contours. Perception 45:315-327.

Pachitariu M, Steinmetz N, Kadir S, Carandini M, and Harris KD (2016) Kilosort: realtime spike-sorting for extracellular electrophysiology with hundreds of channels. BioRxiv 061481

Pan Y, Chen M, Yin J, An X, Zhang X, Lu Y, Gong H, Li W, Wang W (2012) Equivalent representation of real and illusory contours in macaque V4. J Neurosci 32:6760-6770.

Peirce JW (2008) Generating stimuli for neuroscience using PsychoPy. Front Neuroinform 2:10.

Peterhans E, von der Heydt R (1989) Mechanisms of contour perception in monkey visual cortex: II. Contours bridging gaps. J Neurosci 9:1749-1763.

Polley DB, Steinberg EE, Merzenich MM (2006) Perceptual learning directs auditory cortical map reorganization through top-down influences. J Neurosci 26:4970-4982.

Rossant C, Kadir SN, Goodman DFM, Schulman J, Hunter MLD, Saleem AB, Grosmark A, Belluscio M, Denfield GH, Ecker AS, Tolias AS, Solomon S, Buzsaki G, Carandini M, Harris KD (2016) Spike sorting for large, dense electrode arrays. Nat Neurosci 19:634-641.

Schnabel UH, Bossens C, Lorteije JAM, Self MW, Op de Beeck H, Roelfsema PR (2018) Figure-ground perception in the awake mouse and neuronal activity elicited by figure-ground stimuli in primary visual cortex. Sci Rep 8:17800.

Sheth BR, Sharma J, Rao SC, Sur M (1996) Orientation maps of subjective contours in visual cortex. Science 274:2110-2115.

Shobe JL, Claar LD, Parhami S, Bakhurin KI, Masmanidis SC (2015) Brain activity mapping at multiple scales with silicon microprobes containing 1,024 electrodes. J Neurophysiol 114:2043-2052.

von der Heydt R, Peterhans E (1989) Mechanisms of contour perception in monkey visual cortex: I. Lines of pattern discontinuity. J Neurosci 9:1731-1748.

Wang Q, Burkhalter A (2007) Area map of mouse visual cortex. J Comp Neurol 502:339-357.

Wyatte D, Curran T, O'Reilly R (2012) The limits of feedforward vision: recurrent processing promotes robust object recognition when objects are degraded. J Cogn Neurosci 24:2248-2261.

Wyatte D, Jilk DJ, O’Reilly RC (2014) Early recurrent feedback facilitates visual object recognition under challenging conditions. Front Psychol 5:674.

Zhuang J, Ng L, Williams D, Valley M, Li Y, Garrett M, Waters J (2017) An extended retinotopic map of mouse cortex. eLife 6:e18372. 